http://economix.fr

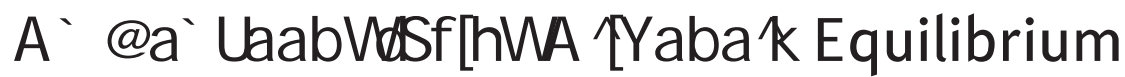 in the Multiple Leader-Follower Game
}




\title{
On Noncooperative Oligopoly Equilibrium in the Multiple Leader-Follower Game
}

\author{
Ludovic A. Julien \\ EconomiX, Université de Paris Ouest-Nanterre La Défense \\ 200 avenue de la République, 92001 Nanterre Cédex, France. \\ E-mail: ludovic.julien@u-paris10.fr
}

Version: February, 1rst 2016

\begin{abstract}
In this paper, we provide new proofs of existence and uniqueness of a Stackelberg market equilibrium for a multiple leader-follower noncooperative oligopoly model in which heterogeneous firms compete on quantities. To this end, we consider a two-step game of perfect and complete information in which many leaders interact strategically with many followers. The Stackelberg market equilibrium constitutes a pure strategy subgame perfect Nash equilibrium of this game. The existence (and uniqueness) problem is complexified in this framework since strategic interactions occur within each partial game but also between both partial games through sequential decisions. Then, to prove existence, we notably provide a new procedure to determine (the conditions under which) the optimal behavior of the followers (may be written) as functions of the leaders' strategy profile only. Some examples outline our procedure and discuss our assumptions.
\end{abstract}

Key Words: Best response functions, existence, uniqueness.

Subject Classification: C61, C62, C72, D43

\section{INTRODUCTION}

Stackelberg competition (1934) portrays a model of a market economy in which strategic firms move sequentially and compete on quantities. In the basic duopoly model one firm (the leader) moves first and makes her decision by taking into consideration the reaction of the other firm (the follower). The leader perfectly knows her rival best response function. The follower can set his own supply according to any possible function of the quantity set by the leader, with the belief that the leader will not counter-react. Similarly, the leader expects the follower to conform to the decisions given by his best response function. A Stackelberg equilibrium (SE thereafter) is a noncooperative equilibrium of a two-step game with perfect and complete information where the players are the firms, the strategies are their production decisions, and their payoffs are their profit functions. A SE constitutes a pure strategy subgame perfect Nash equilibrium (SPNE). Existence and uniqueness of SE are studied by Robson (1990) in the $T$-stage model of Boyer and Moreaux (1986), with one monopoly firm per stage. Alj et al. (1988) and Freiling et al. (2001) show existence in differential duopoly games. The common feature of these models is the leader behaves as a monopolist by using the well-defined follower's best response. But the existence (and uniqueness) problem is complexified with multiple leaders and followers as strategic interactions also occur within step of the game. As a consequence, in the presence of heterogeneous followers, the best responses could not be well-defined. This problem is the starting point of this paper. 
This paper deals with the existence and uniqueness of a SE in a multiple leaderfollower model. To this end, we consider an extension of the two-stage deterministic and static noncooperative multiple leader-follower oligopoly model introduced by Sherali (1984). There are several leaders and followers who compete on quantities to sell one homogeneous divisible product. This hierarchical model consists of two Cournot competitions encompassed by a Stackelberg competition. This model is thus described by a two-step game which embodies two simultaneous move partial games. Indeed, the leaders play a two-step game with the followers, but the leaders (the followers) play together a simultaneous move game. We assume the timing of positions is given. Information is assumed to be perfect and complete. Thus, we look for pure strategy SPNE. In addition, we focus on the case where pure strategies are substitutes. Existence and uniqueness problems are complexified in this framework since strategic interactions occur within each partial game but also between both partial games through sequential decisions. One difficulty stems from the fact that any leader faces many followers optimal decision mappings. In a decentralized economy firms determine their optimal decisions without central coordination device: each individual firm acts independently and without communication with any of the others (see notably Johansen (1982) and Daughety (2009)). Therefore, the resulting followers' (leaders') optimal decision mappings might be mutually inconsistent. More specifically, the followers' optimal decisions must be internally consistent to solve the game. Under this consistency requirement, the set of optimal decision mappings of followers can determine the best responses as functions of the strategies of leaders only. This problem is eluded in the literature and deserves more careful study. One novelty of our paper is that we provide a simple consistency criterion which determines the conditions under which such best response functions may be obtained. Our criterion also creates a logical link between the two partial games to study existence and uniqueness issues. Indeed, our existence and uniqueness proofs stem from a consistency (sufficient) condition among followers' decision mappings, each function being based on decentralized optimizing behavior.

Existence and uniqueness have already been explored in the multiple leaderfollower model. Sherali (1984) shows existence and uniqueness with identical convex costs for leaders, and states some results under the assumptions of linear demand either with linear or quadratic costs (see also Ehrenmann (2004)). Sherali's model constitutes an extension of the seminal paper of Murphy et al. (1983) which covers the case of many followers who interact with one leader. In their model the authors provide a characterization of the SE and an algorithm to compute it. They state a Theorem 1 which gives the properties of the aggregate best response of the followers as a function of the leader's strategy. This determination stems from a family of optimization programs for the followers conceived as a price function perturbed by the supply of the leader. They show this aggregate function is convex, and then, study the leader's problem. But they do not study the conditions under which the followers' optimal decisions are mutually consistent. In the same vein, Tobin (1992) provides an efficient algorithm to find a unique SE by parameterizing the price function by the leader's strategy. Some strong assumptions on the thricedifferentiability of the price function and cost function of the leader profit function are made. Following De Wolf and Smeers (1997) who extend Murphy et al. (1983), De Miguel and $\mathrm{Xu}$ (2009) extend (1984) to uncertainty with stochastic market demand. Unlike Sherali (1984) they allow costs to differ across leaders. Nevertheless, to show the concavity of the expected profit of any leader, they assume that the follower aggregate best response is convex. But as this assumption does not always 
hold, they must resort to a linear demand. Fukushima and Pang (2005), Yu and Wang (2008), and He et al. (2015) prove existence of an equilibrium point of a finite game with two leaders and several followers without specifying the assumptions made on demand and costs. In this paper, we consider a simple deterministic version of the hierarchical model without strategic complementarities.

Our contribution to the literature is twofold. First, existence and uniqueness of a SE are obtained under weaker assumptions on cost and demand functions. The demand function is not specific and firms may not bear the same costs. In addition, by contrast with Sherali (1984), Ehrenmann (2004) and De Miguel and Xu (2009), some firms (not all) may have nonconvex costs. Second, we provide a new procedure to characterize a SE, and thereby to prove its existence and its uniqueness. A $\mathrm{SE}$ is the outcome of a decentralized mechanism through which seperate rational firms interact strategically. Indeed, our model is made up of two partial games encompassed by an entire game. Within each partial game the behavior of any leader (follower) is based on a separate maximization program. Therefore, the compatibility among individual decisions should be satisfied within each step, but also between both steps of the game. The link between the two partial games is studied through a system of equations which specifies a mutual consistency condition which is critical to derive followers' best responses. To this end, we provide a criterion to obtain the best responses from which we derive the effective demand which addresses to any leader. This criterion works under some regularity conditions linked to differentiability.

Within this framework we obtain the following results. First, we determine the optimal decision of any follower as a function of the strategy profiles of the other followers and of the leaders. We show such functions are decreasing, with bounded partial derivatives. Then, we consider the existence of the best response functions of followers. To this end, we give a criterion to test the consistency of the system of equations which allows the determination of best responses. Hence, if the Jacobian matrix of followers best responses is of full rank, then, each follower's best response may be written as a function of leaders' strategy profile. Then, we consider any leader's problem when she faces the effective demand, that is, the price as a function of leaders' strategy profile only. We also study the optimal decisions of leaders. Third, we consider the entire game and show existence and uniqueness of an active SE, i.e., an equilibrium with strictly positive strategies. Existence is shown by using some fixed point argument. Uniqueness is obtained by using some mild assumptions on costs and demand functions. The advantages of our approach are threefold. First, we provide a characterization of the strategic equilibrium which brings into light a consistency criterion. The possibility to solve the game depends on whether our criterion is satisfied. If the criterion holds, our finite extensive form game of perfect information may have a unique pure strategy SPNE. Second, we make some general assumptions on costs and demand functions. Third, our procedure puts forward the beliefs of leaders: by construction of a SE they know the reactions of the followers through the slope of the aggregate best response. Indeed, any leaders' markup and Lerner index, derived from the optimality conditions, are expressed in terms of this slope. Thus, the behavior of leaders deserve also to be analyzed in detail.

The remaining of the paper is organized as follows. In section 2 we present the model. Section 3 is devoted to a definition of the SE which relates to rational behavior and to our consistency criterion. Existence and uniqueness are studied in Section 4. Section 5 provides some examples to discuss our assumptions and to illustrate our criterion. In section 6 we conclude. 


\section{THE MODEL}

The model is described in three steps. First, we set up the framework and fix some notations. Second, we give two assumptions and we discuss each of them. Third, we complete the description of the model with the associated game.

\subsection{Framework and notations}

Consider an oligopoly market with a single divisible homogeneous product. There is a finite set of firms $\mathcal{F}$ which embodies two types of firms labeled $F$ and $L$. Thus, the set $\mathcal{F}$ partitions into two subsets $\mathcal{F}_{L}=\left\{1, \ldots, n_{L}\right\}$ and $\mathcal{F}_{F}=\left\{1, \ldots, n_{F}\right\}$, with $\mathcal{F}_{L} \cup \mathcal{F}_{F}=\mathcal{F}$ and $\mathcal{F}_{L} \cap \mathcal{F}_{F}=\varnothing$. We consider $\left|\mathcal{F}_{L}\right| \geqslant 1$ and $\left|\mathcal{F}_{F}\right| \geqslant 1$, where $|A|$ denotes the cardinality of the set $A$. Firms of type $L$ are leaders, while firms of type $F$ are followers. Leaders are indexed by $i$ and followers are indexed by $j$.

We adopt the following notational conventions. Let $\mathbf{x} \in \mathbb{R}_{+}^{n}$. Then, $\mathbf{x} \geq \mathbf{0}$ means $x_{i} \geqslant 0, i=1, \ldots, n ; \mathbf{x}>\mathbf{0}$ means there is some $i$ such that $x_{i}>0$, with $\mathbf{x} \neq \mathbf{0}$, and $\mathbf{x}>>\mathbf{0}$ means $x_{i}>0$ for all $i, i=1, \ldots, n$. Let $\mathbf{x}_{L}=\left(x_{L}^{1}, \ldots, x_{L}^{i}, \ldots, x_{L}^{n_{L}}\right)$ be a strategy profile of leaders, and $\mathbf{x}_{F}=\left(x_{F}^{1}, \ldots, x_{F}^{j}, \ldots, x_{F}^{n_{F}}\right)$ be a strategy profile of followers, where $x_{L}^{i}$ and $x_{F}^{j}$ represent respectively the supply of leader $i \in \mathcal{F}_{L}$, and of follower $j \in \mathcal{F}_{F}$. In addition, let $\mathbf{x}_{L}^{-i}=\left(x_{L}^{1}, \ldots, x_{L}^{i-1}, x_{L}^{i+1}, \ldots, x_{L}^{i}\right)$ and $\mathbf{x}_{F}^{-j}=\left(x_{F}^{1}, \ldots, x_{F}^{j-1}, x_{F}^{j+1}, \ldots, x_{F}^{j}\right)$. We sometimes consider $X_{L} \equiv \sum_{i \in \mathcal{F}_{L}} x_{L}^{i}$ and $X_{F} \equiv \sum_{j \in \mathcal{F}_{F}} x_{F}^{j}$. In addition, let $X_{L}^{-i} \equiv \sum_{-i \in \mathcal{F}_{L}} x_{L}^{-i}$, with $X_{L}^{-i}=X_{L}-x_{L}^{i}$, and $X_{F}^{-j} \equiv \sum_{-j \in \mathcal{F}_{F}} x_{F}^{-j}$, with $X_{F}^{-j}=X_{F}-x_{F}^{j}$. Let $f$ be a function defined by $f: A \subseteq \mathbb{R}^{n} \rightarrow B \subseteq \mathbb{R}$, with $\mathbf{z} \longmapsto f(\mathbf{z})$. The partial derivative of $f$ with respect to $z_{i}$ will be denoted by $\frac{\partial f}{\partial z_{i}}, i=1, \ldots, n$. When $n=1$ the derivative is $\frac{d f}{d z}$. The partial derivative of $f$ with respect to $z_{i}$ at $z_{i}=\bar{z}_{i}$ is denoted by $\frac{\partial f}{\partial z_{i}}\left(\bar{z}_{i}\right)$. The same holds with $\frac{d f}{d z}(\bar{z})$. A $m$ dimensional vector function $\mathbf{F}$ is defined by $\mathbf{F}$ : $A \subseteq \mathbb{R}^{n} \rightarrow B \subseteq \mathbb{R}^{m}$, with $\mathbf{F}(\mathbf{z})=\left(f^{1}(\mathbf{z}), \ldots, f^{j}(\mathbf{z}), \ldots, f^{m}(\mathbf{z})\right)$. The notation $\mathbf{z}(\mathbf{y})$, where $\mathbf{y} \in \mathbb{R}^{k}$, means that each $z_{i}$ is a function of $\mathbf{y}$. The Jacobian matrix of $\mathbf{F}(\mathbf{z})$ with respect to $\mathbf{z}$ at $\overline{\mathbf{z}}$ will be denoted by $\mathcal{J}_{\mathbf{F}}(\overline{\mathbf{z}})$, with $\mathcal{J}_{\mathbf{F}}(\overline{\mathbf{z}})=\left[\frac{\partial\left(f^{1}, \ldots, f^{j}, \ldots, f^{m}\right)}{\partial\left(z^{1}, \ldots, z^{i}, \ldots, z^{n}\right)}\right]$. Let $\left|\mathcal{J}_{\mathbf{F}}(\overline{\mathbf{z}})\right|$ be the determinant of $\mathcal{J}_{\mathbf{F}}$ at $\overline{\mathbf{z}}$. Finally, when the distinction matters, if we partition $\mathbf{z}$ in such a way $\mathbf{z}=(\mathbf{x}, \mathbf{y})$, then $\mathcal{J}_{\mathbf{F}_{\mathbf{x}}}(\overline{\mathbf{z}})$ is the Jacobian matrix of $\mathbf{F}(\mathbf{z})$ at $\overline{\mathbf{z}}$ when the differentiation is partial and made with respect to $\mathbf{x}$ only. The corresponding determinant is denoted by $\left|\mathcal{J}_{\mathbf{F}_{\mathbf{x}}}(\overline{\mathbf{z}})\right|$.

\subsection{Assumptions: statement and discussion}

We now make some assumptions regarding the demand side and the supply side of the market. There is a continuous market demand function $D(p)$ which balances the aggregate supply $X$, with $X \equiv X_{L}+X_{F}$. It represents the maximum price the consumers are willing to pay to buy the quantity $X$ as well the minimum price the firms are willing to charge to sell the corresponding production $X$. Indeed, let $X \longmapsto p(X)=D^{-1}(X)$ be the market inverse demand function. We make the following set of assumptions regarding $p(X)$, which we designate as Assumption 1. 
Assumption 1. The price function $p(X)$ satisfies the following:

(1a) $p(X) \geqslant 0$ for all $X \geqslant 0$, with $p(X) \in C^{2}\left(\mathbb{R}_{++}\right)$;

(1b) $\frac{d p(X)}{d X}<0$ for $X \geqslant 0$;

(1c) $\forall x \geqslant 0, \frac{d p(X)}{d X}+k x \frac{d^{2} p(X)}{(d X)^{2}} \leqslant 0$, where $k>0$.

(1a) says that the inverse demand function $p(X)$ is positively valued, and it may or may not intersect both the quantity axis and the price axis. We do not preclude that the price function intersects only one of the two axis. For instance, if $p(X)=\frac{1}{X}-1$ with $n_{F}=n_{L}=1$ yields $x_{L}=\frac{1}{4}$ and $x_{F}=\frac{1}{4}$, with $\bar{X}=1$. If it would intersect only the price axis since we had $\lim _{X \rightarrow 0} p(X)=\bar{p}>0$ (for instance: $p(X)=\sqrt{1-X}$ with $\left|\mathcal{F}_{F}\right|=\left|\mathcal{F}_{L}\right|=1$ yields $x_{L}=\frac{1}{3}$ and $x_{F}=\frac{1}{9}$ ). Therefore, (1a) does not impose too stringent property on the market demand function: it may be strictly concave (convex) or linear, without imposing some boundary conditions. (1a) also says that $p(X)$ is well-behaved: it is twice continuously differentiable on the open set $\mathbb{R}_{++}$. This assumption will be useful notably to characterize the optimal behavior in a Stackelberg market in Section 3.

(1b) is obvious.

(1c) stipulates that marginal revenue for any single firm is a decreasing function of total industry output. This formulation deserves two comments. First, we do not impose $\frac{d^{2} p(X)}{(d X)^{2}} \leqslant 0$, so we do not preclude (strictly) convex market demand functions. Second, our formulation of the decreasing marginal revenue hypothesis embodies the parameter $k$. For any follower firm, we have $k=1$, as in the Cournot model (Hahn (1962), Okugushi (1976)). However, for any leader firm $k$ may be generally different from unity unless leaders behave as followers (see Section 3). Our formulation puts forward an important feature of Stackelberg competition which explicitly takes into account leader firms have perfect information regarding the optimal reactions of followers to change in their strategies (Julien (2011)).

Each firm bears some costs. Cost functions satisfy the following set of assumptions, which we designate by Assumption 2 .

Assumption 2. The cost function $c^{h}\left(x^{h}\right), h \in \mathcal{F}$, satisfies the following:

(2a) $\forall h \in \mathcal{F}, c^{h}\left(x^{h}\right) \geqslant 0$ for all $x \geqslant 0, c^{h}\left(x^{h}\right) \in C^{2}\left(\mathbb{R}_{++}\right)$;

(2b) $\forall h \in \mathcal{F}, \frac{d^{2} c^{h}\left(x^{h}\right)}{\left(d x^{h}\right)^{2}}-k \frac{d p(X)}{d X}>0, k>0$ for each $x^{h}>0$;

(2c) $p(0)>\max \left\{\frac{d c^{h}}{d x^{h}}(0), h \in \mathcal{F}\right\}$, and there exists $X^{*}>0$ large enough for which $\frac{d c^{h}}{d x^{h}}\left(X^{*}\right) \geqslant p\left(X^{*}\right)$, for all $X \geqslant X^{*}, \forall h \in \mathcal{F}$.

Let $c_{L}^{i}\left(x_{L}^{i}\right)$, for each $i \in \mathcal{F}_{L}$, and $c_{F}^{j}\left(x_{F}^{j}\right)$, for each $j \in \mathcal{F}_{F}$.

(2a) stipulates that the cost functions are positive and twice continuously differentiable on the open set $\mathbb{R}_{++}$.

$(2 \mathrm{~b})$ requires that the marginal cost rises faster than the decrease in the average revenue (the price). Let us notice costs may be different and need not be convex for all firms, but it precludes concave costs for all firms. In the presence of increasing returns, firms should supply nothing. For instance, if $\left|\mathcal{F}_{F}\right|=\left|\mathcal{F}_{L}\right|=1$, with $p(X)=\frac{4}{X}$, and $c_{F}\left(x_{F}\right)=\ln \left(1+x_{F}\right)$ and $c_{L}\left(x_{L}\right)=2 \sqrt{x_{L}}$, then the market outcome is the trivial solution, i.e., $x_{L}=x_{F}=0$. Hence, market outcomes with positive supplies require smooth costs. This condition is not stated exactly in the same way for leaders and for followers since leaders know the reactions of followers. 
(2c) stipulates two things. First, the maximum price exceeds the higher marginal costs. It is assumed in all models; in particular the standard linear model with linear demand $p(X)=a-b X$ and constant marginal costs $c_{1}$ and $c_{2}$, with $c_{1}>c_{2}$, considers $a>c_{1}$. Second, industry supply is bounded if there is some large output level for which marginal costs exceeds any firm's revenue, leading to negative marginal profits. Hence, to prevent high production supply, we assume no firm wishes to produce such a large quantity. Therefore, there is an upper bound on the production set, which compactifies the set of possible supply (see Murphy et al. (1983)). Thus, (2c) is a weakening of the usual assumption made on inverse market demand; we do not assume there is some finite large quantity $\bar{X}<\infty$ for which $p(X)=0$, for all $X \geqslant \bar{X}$ (Frank and Quandt (1963), Novshek (1985)).

The profit functions $\pi_{L}^{i}($.$) of firm i$ and $\pi_{F}^{j}($.$) of firm j$ may be written:

$$
\begin{gathered}
\pi_{L}^{i}\left(x_{L}^{i}, \mathbf{x}_{L}^{-i}, \mathbf{x}_{F}\right)=p(X) x_{L}^{i}-c_{L}^{i}\left(x_{L}^{i}\right), i \in \mathcal{F}_{L} \\
\pi_{F}^{j}\left(x_{F}^{j}, \mathbf{x}_{F}^{-j}, \mathbf{x}_{L}\right)=p(X) x_{F}^{j}-c_{F}^{j}\left(x_{F}^{j}\right), j \in \mathcal{F}_{F} .
\end{gathered}
$$

Proposition 1. Let Assumption 1 and (2a)-(2b) be satisfied. Then, the profit function (2) is strictly concave with respect to $x_{F}^{j}$ given $\mathbf{x}_{F}^{-j}$ and $\mathbf{x}_{L}$.

Proof. Differentiating partially twice (2) with respect to $x_{F}^{j}$, and using (1c) and (2b) with $k=1$, we deduce $\frac{\partial^{2} \pi_{F}^{j}}{\left(\partial x_{F}^{j}\right)^{2}}=2 \frac{d p(X)}{d X}+x_{F}^{j} \frac{d^{2} p(X)}{(d X)^{2}}-\frac{d^{2} c_{F}^{j}\left(x_{F}^{j}\right)}{\left(d x_{F}^{j}\right)^{2}}<0$.

Remark 1. A rather more complicated expression than the preceding one holds for any leader (see Section 4, Remark 2).

\subsection{The Stackelberg game}

To this economy we associate a game $\boldsymbol{\Gamma}$. The players are the firms, the strategies are the production decisions, and the payoffs are the profits. Let $\mathcal{S}_{L}^{i}=$ $\left\{x_{L}^{i} \in \mathbb{R}_{+}: 0 \leqslant x_{L}^{i} \leqslant X^{*}\right\}$ and $\mathcal{S}_{F}^{j}=\left\{x_{F}^{j} \in \mathbb{R}_{+}: 0 \leqslant x_{F}^{j} \leqslant X^{*}\right\}$ be the strategy sets of leader $i \in \mathcal{F}_{L}$ and follower $j \in \mathcal{F}_{F}$ respectively. The supply $x_{L}^{i}$ (resp. $x_{F}^{j}$ ) represents the pure strategy of leader $i \in \mathcal{F}_{L}$ (follower $j \in \mathcal{F}_{F}$ ). A strategy profile will be represented by the vector $\left(\mathbf{x}_{L}, \mathbf{x}_{F}\right)$, with $\left(\mathbf{x}_{L}, \mathbf{x}_{F}\right) \in \prod_{i \in \mathcal{F}_{L}} \mathcal{S}_{L}^{i} \times \prod_{j \in \mathcal{F}_{F}} \mathcal{S}_{F}^{j}$. The corresponding payoffs are given by $\pi_{L}^{i}, i \in \mathcal{F}_{L}$, and $\pi_{F}^{j}, j \in \mathcal{F}_{F}$.

This game displays two stages of decisions and no discounting. We also assume the timing of positions as given. Thus, this hierarchical model consists of two Cournot competitions encompassed by a Stackelberg competition. Indeed, the $n_{L}$ leaders play a two-step game with the $n_{F}$ followers, but the leaders (the followers) play a simultaneous moves game. Finally, information is assumed to be complete and perfect. It notably implies that any leader perfectly knows the followers' behavior. Any leader is able to perceive the reactions of any follower. Perfect information also implies that, for any follower, each information set is a single decision node. In addition, in each decision node, any follower makes an optimal choice, so sequential rationality prevails. As sequential rationality is common knowledge, the game is solved by backward induction, considering first the optimal strategic decisions of the followers, and then the optimal strategic choices of the leaders. 


\section{OPTIMAL BEHAVIOR}

In contrast with the duopoly game the behavior of firms are complexified in this model. First, given a feasible strategy profile for the leaders $\mathbf{x}_{L}$, each follower will determine her optimal decision as a mapping whose arguments are the strategies of all other followers and the strategies of all leaders. But the followers optimal decisions must be consistent. This consistency means that each optimal decision mapping may be reduced to a best response function of the leaders's strategies only. The following subsections provide three Lemmas. Lemmas 1 and 3 characterize followers and leaders behavior respectively, while Lemma 2 provides a consistency condition to determine the effective demand which addresses to any leader.

\subsection{The followers}

Definition 1. Let $\phi^{j}: \prod_{-j \in \mathcal{F}_{F}} \mathcal{S}_{F}^{-j} \times \prod_{i \in \mathcal{F}_{L}} \mathcal{S}_{L}^{i} \rightarrow \mathcal{S}_{F}^{j}$, with $\phi^{j}\left(\mathbf{x}_{F}^{-j}, \mathbf{x}_{L}\right)=\left\{x_{F}^{j} \in\right.$ $\left.\mathcal{S}_{F}^{j}: x_{F}^{j} \in \arg \max \pi_{F}^{j}\left(x_{F}^{j}, \mathbf{x}_{F}^{-j}, \mathbf{x}_{L}\right)\right\}$ be follower $j$ 's optimal decision, $j \in \mathcal{F}_{F}$.

Lemma 1. Let the function $p($.$) satisfies Assumption 1, and the functions c_{F}^{j}($.$) ,$ $j \in \mathcal{F}_{F}$, satisfy Assumption 2. Then, for each $j \in \mathcal{F}_{F}$, the mapping $\phi^{j}\left(\mathbf{x}_{F}^{-j}, \mathbf{x}_{L}\right)$ is well defined, point-valued (a function) and continuously differentiable.

Proof. The mapping $\phi^{j}\left(\mathbf{x}_{L}, \mathbf{x}_{F}^{-j}\right)$ is well defined. The program of follower $j$ consists in maximizing $\pi_{F}^{j}\left(x_{F}^{j}, \mathbf{x}_{F}^{-j}, \mathbf{x}_{L}\right)$, a strictly concave function with respect to $x_{F}^{j}$ (by Proposition 1), subject to $x_{F}^{j} \in\left[0, X^{*}\right]$, a nonempty and compact (convex) set (by $(2 \mathrm{c})$ ). As the profit function is strictly concave it is continuous. Then, from the Weierstrass Theorem, the set $\arg \max \left\{\pi_{F}^{j}\left(x_{F}^{j}, . ..\right): x_{F}^{j} \in\left[0, X^{*}\right]\right\}$ is nonempty, so there exists $\phi^{j}: \prod_{-j \neq j} \mathcal{S}_{F}^{-j} \times \prod_{i} \mathcal{S}_{L}^{i} \rightarrow \mathcal{S}_{F}^{j}$, such that $x_{F}^{j}=\phi^{j}\left(\mathbf{x}_{F}^{-j}, \mathbf{x}_{L}\right), j \in \mathcal{F}_{F}$. In addition, from Proposition 1 , we deduce $\phi^{j}\left(\mathbf{x}_{F}^{-j}, \mathbf{x}_{L}\right)$ is point valued. We now characterize follower $j$ 's optimal behavior. Let $\mathcal{L}$ be the Lagrangian and $\lambda^{j}$ and $\mu^{j}$ the nonnegative Lagrange multipliers. Then, given $\mathbf{x}_{L}$, with $\mathbf{x}_{L} \in \prod_{i} \mathcal{S}_{L}^{i}$, follower $j$ 's optimal decision is the solution to:

$$
\max \mathcal{L}\left(x_{F}^{j}, \mathbf{x}_{F}^{-j}, \mathbf{x}_{L}, \lambda^{j}, \mu^{j}\right):=\pi_{F}^{j}\left(x_{F}^{j}, \mathbf{x}_{F}^{-j}, \mathbf{x}_{L}\right)+\lambda^{j} x_{F}^{j}+\mu^{j}\left(X^{*}-x_{F}^{j}\right), j \in \mathcal{F}_{F} .
$$

The Kuhn-Tucker conditions may be written:

$$
\begin{aligned}
\frac{\partial \mathcal{L}}{\partial x_{F}^{j}} & =\frac{\partial \pi_{F}^{j}}{\partial x_{F}^{j}}+\lambda^{j}-\mu^{j}=0, j \in \mathcal{F}_{F} \\
\lambda^{j} & \geqslant 0, x_{F}^{j} \geqslant 0, \text { with } \lambda^{j} x_{F}^{j}=0 \\
\mu^{j} & \geqslant 0,\left(X^{*}-x_{F}^{j}\right) \geqslant 0, \text { with } \mu^{j}\left(X^{*}-x_{F}^{j}\right)=0,
\end{aligned}
$$

where $\frac{\partial \pi_{F}^{j}}{\partial x_{F}^{j}}=p(X)+x_{F}^{j} \frac{d p(X)}{d X}-\frac{d c_{F}^{j}\left(x_{F}^{j}\right)}{d x_{F}^{j}}$. From (1a), we have either $\phi^{j}\left(\mathbf{x}_{F}^{-j}, \mathbf{x}_{L}\right)=0$ or $\phi^{j}\left(\mathbf{x}_{F}^{-j}, \mathbf{x}_{L}\right)>0$. Therefore, if $x_{F}^{j}>0$, then $\lambda^{j}=0$, where $x_{F}^{j}$ is the solution to the equation $p(X)+x_{F}^{j} \frac{d p(X)}{d X}-\frac{d c_{F}^{j}\left(x_{F}^{j}\right)}{d x_{F}^{j}}=\mu^{j}$, which yields $\phi^{j}\left(\mathbf{x}_{F}^{-j}, \mathbf{x}_{L}\right)>0$. In addition, if $\mu^{j}>0$, then $x_{F}^{j}=\phi^{j}(\mathbf{0}, \mathbf{0})=X^{*}$, while if $\mu^{j}=0$, then $\phi^{j}\left(\mathbf{x}_{F}^{-j}, \mathbf{x}_{L}\right) \in$ $\left(0, X^{*}\right)$. Now, if $\lambda^{j}>0$, then $x_{F}^{j}=0$, which means that $\phi^{j}\left(\mathbf{x}_{F}^{-j}, \mathbf{x}_{L}\right)=0$ and 
$\mu^{j}=0$ since $x_{F}^{j}<X^{*}$. Then, either $\phi^{j}\left(\mathbf{x}_{F}^{-j}, \mathbf{x}_{L}\right)>0$ whenever $x_{F}^{j} \in\left(0, X^{*}\right]$ or $\phi^{j}\left(\mathbf{x}_{F}^{-j}, \mathbf{x}_{L}\right)=0, j \in \mathcal{F}_{F}$. Then, $\phi^{j}\left(\mathbf{x}_{F}^{-j}, \mathbf{x}_{L}\right) \geqslant 0, j \in \mathcal{F}_{F}$.

The function $\phi^{j}\left(\mathbf{x}_{F}^{-j}, \mathbf{x}_{L}\right)$ is continuously differentiable. Using Proposition 1, then, from the Berge Maximum Theorem (1963), we deduce $x_{F}^{j}=\phi^{j}\left(\mathbf{x}_{F}^{-j}, \mathbf{x}_{L}\right)$, $j \in \mathcal{F}_{F}$, is $C^{1}$ on $\prod_{-j \neq j} \mathcal{S}^{-j} \times \prod_{i} \mathcal{S}^{i}$.

Proposition 2. Let $\phi: \prod_{i \in \mathcal{F}_{L}} \mathcal{S}^{i} \times \prod_{j \in \mathcal{F}_{F}} \mathcal{S}^{j} \rightarrow \prod_{j \in \mathcal{F}_{F}} \mathcal{S}^{j}$ be the $n_{F}$ dimensional vector function defined by $\phi=\left(\phi^{1}, \ldots, \phi^{j}, \ldots, \phi^{n_{F}}\right)$. Consider the Jacobian matrices $\mathcal{J}_{\phi_{\mathbf{x}_{F}}}\left(\overline{\mathbf{x}}_{F}, \overline{\mathbf{x}}_{L}\right)=\left[\frac{\partial\left(\phi^{1}, \ldots, \phi^{j}, \ldots, \phi^{n} F\right)}{\partial\left(x_{F}^{1}, \ldots, x_{F}^{j}, \ldots, x_{F}^{n}\right)}\right]$ and $\mathcal{J}_{\phi_{\mathbf{x}_{L}}}\left(\overline{\mathbf{x}}_{F}, \overline{\mathbf{x}}_{L}\right)=\left[\frac{\partial\left(\phi^{1}, \ldots, \phi^{j}, \ldots, \phi^{n} F\right)}{\partial\left(x_{L}^{1}, \ldots, x_{L}^{i}, \ldots, x_{L}^{n_{L}}\right)}\right]$. Then, $-\mathcal{I}_{\left(n_{F}, n_{F}\right)}<<\mathcal{J}_{\phi_{\mathbf{x}_{F}}}\left(\overline{\mathbf{x}}_{F}, \overline{\mathbf{x}}_{L}\right) \leq \mathbf{0}_{\left(n_{F}, n_{F}\right)}$ and $-\mathcal{I}_{\left(n_{F}, n_{L}\right)}<<\mathcal{J}_{\phi_{\mathbf{x}_{L}}}\left(\overline{\mathbf{x}}_{F}, \overline{\mathbf{x}}_{L}\right) \leq$ $\mathbf{0}_{\left(n_{F}, n_{L}\right)}$, where $\mathbf{0}$ and $\mathcal{I}$ are the zero matrix and the unit matrix respectively.

Proof. See Appendix A.

Proposition 2 notably states that each follower's optimal decision function is decreasing in the strategy of each other follower and in the strategy of each leader.

\subsection{The best responses: a consistency criterion}

Each leader has perfect information on the optimal decisions. But before considering the problem of any leader we must check that the system of optimal decisions is internally consistent: each follower's optimal decision mapping may be expressed as a function of the leaders's strategies only. To this end, we provide a consistency criterion which determines each optimal decision as a function of the strategy profile of the leaders. Indeed, any leader firm is then able to solve the system of optimal decision mappings to obtain the best responses as functions of his own strategy and of the other leaders' strategies. It leads to the effective demand (the price function as a mapping of the leaders' strategic supplies only) which addresses to any leader. Otherwise, if the criterion is not satisfied, we cannot determine the individual best responses. Example 3 in Section 5 illustrates this fact.

Let the system of $n_{F}$ simultaneous equations with $n_{F}$ unknowns $x_{F}^{j}$ and $n_{L}$ parameters be given by:

$$
x_{F}^{j}-\phi^{j}\left(\mathbf{x}_{F}^{-j}, \mathbf{x}_{L}\right)=0, j \in \mathcal{F}_{F} .
$$

These $n_{F}$ equations taken together consist in a system of equations, whose solution, if it exists, provides best responses.

Definition 2. Let $\varphi^{j}: \prod_{i \in \mathcal{F}_{L}} \mathcal{S}^{i} \rightarrow \mathcal{S}^{j}$, with $x_{F}^{j}=\varphi^{j}\left(\mathbf{x}_{L}\right)$, be the best response of follower $j, j \in \mathcal{F}_{F}$.

To introduce our criterion define the function $\Phi^{j}: \prod_{j \in \mathcal{F}_{F}} \mathcal{S}_{F}^{j} \times \prod_{i \in \mathcal{F}_{L}} \mathcal{S}_{L}^{i} \rightarrow \mathcal{S}^{j}$, with $\Phi^{j}\left(\mathbf{x}_{F}, \mathbf{x}_{L}\right) \equiv x_{F}^{j}-\phi^{j}\left(\mathbf{x}_{F}^{-j}, \mathbf{x}_{L}\right), j \in \mathcal{F}_{F}$. Let $\mathbf{\Phi}: \prod_{j \in \mathcal{F}_{F}} \mathcal{S}_{F}^{j} \times \prod_{i \in \mathcal{F}_{L}} \mathcal{S}_{L}^{i} \rightarrow \prod_{j \in \mathcal{F}_{F}} \mathcal{S}_{F}^{j}$ the $n_{F}$ dimensional vector function defined by $\boldsymbol{\Phi}=\left(\Phi^{1}, \ldots, \Phi^{j}, \ldots, \Phi^{n_{F}}\right)$. Consider the $n_{F}$-dimensional vector equation $\mathbf{\Phi}\left(\mathbf{x}_{F}, \mathbf{x}_{L}\right)=\mathbf{0}$. Let $\mathcal{J}_{\boldsymbol{\Phi}_{\mathbf{x}_{F}}}\left(\overline{\mathbf{x}}_{F}, \overline{\mathbf{x}}_{L}\right)$ be the $\left(n_{F}, n_{F}\right)$ square matrix formed by all partial derivatives of $\Phi^{j}$ with respect to $\mathbf{x}_{F}$ at a point $\left(\overline{\mathbf{x}}_{F}, \overline{\mathbf{x}}_{L}\right)$. The next lemma provides a sufficient condition for the existence (and continuous differentiability) of such best responses. So, Lemma 2 provides a criterion to determine locally the best responses. 
Lemma 2. If $\left|\mathcal{J}_{\boldsymbol{\Phi}_{\mathbf{x}_{F}}}\left(\overline{\mathbf{x}}_{F}, \overline{\mathbf{x}}_{L}\right)\right|>0$, then, for each $j \in \mathcal{F}_{F}$, there exists $\varphi^{j}$ : $\prod_{i \in \mathcal{F}_{L}} \mathcal{S}_{L}^{i} \rightarrow \mathcal{S}_{F}^{j}$, with $x_{F}^{j}=\varphi^{j}\left(\mathbf{x}_{L}\right)$. Moreover, for each $j \in \mathcal{F}_{F}, \varphi^{j} \subset C^{1}\left(\mathbb{R}_{++}\right)$.

Proof. See Appendix B. I

Proposition 3. Let $\varphi^{j}: \prod_{i \in \mathcal{F}_{L}} \mathcal{S}_{L}^{i} \supset \mathcal{U}_{\left(\overline{\mathbf{x}}_{L}\right)} \rightarrow \mathcal{S}_{F}^{j}$, with $x_{F}^{j}=\varphi^{j}\left(\mathbf{x}_{L}\right), j \in \mathcal{F}_{F}$. Consider $\mathcal{J}_{\boldsymbol{\varphi}}\left(\overline{\mathbf{x}}_{L}\right)=\left[\frac{\partial\left(\varphi^{1}, \ldots, \varphi^{j}, \ldots, \varphi^{n_{F}}\right)}{\partial\left(x_{L}^{1}, \ldots, x_{L}^{i}, \ldots, x_{L}^{n_{L}}\right)}\right]$. Then, $-\mathcal{I}_{\left(n_{F}, n_{L}\right)}<<\mathcal{J}_{\boldsymbol{\varphi}}\left(\mathbf{x}_{L}\right) \leq \mathbf{0}_{\left(n_{F}, n_{L}\right)}$.

Proof. See Appendix C. ।

\subsection{The leaders}

In the second step each leader knows how the market price is affected by the followers' reactions. This information is transmitted through the effective demand which adresses to her, i.e., $p\left(x_{L}^{i}+X_{L}^{-i}+\sum_{j} \varphi^{j}\left(\mathbf{x}_{L}\right)\right)$.

Definition 3. Let leader $i$ 's optimal decision be defined by $\psi^{i}: \prod_{-i \in \mathcal{F}_{L}} \mathcal{S}_{L}^{-i} \rightarrow$ $\mathcal{S}_{L}^{i}$, with $\psi^{i}\left(\mathbf{x}_{L}^{-i}\right)=\left\{x_{L}^{i} \in \mathcal{S}_{L}^{i}: x_{L}^{i} \in \arg \max \pi_{L}^{i}\left(x_{L}^{i}, \mathbf{x}_{L}^{-i}, \boldsymbol{\varphi}\left(\mathbf{x}_{L}\right)\right)\right\}, i \in \mathcal{F}_{L}$.

Lemma 3. Let the function $p($.$) satisfies Assumption 1$ and the functions $c_{L}^{i}($.$) ,$ $i \in \mathcal{F}_{L}$, and $c_{F}^{j}(),. j \in \mathcal{F}_{F}$ satisfy Assumption 2. Then, for each $i \in \mathcal{F}_{L}$, the mapping $\psi^{i}\left(\mathbf{x}_{L}^{-i}\right)$ is well defined, point-valued (a function) and continuous on $\prod_{-i} \mathcal{S}_{L}^{-i}$.

Proof. The mapping $\psi^{i}\left(\mathbf{x}_{L}^{-i}\right)$ is well defined. Given $\varphi^{j}: \prod_{i} \mathcal{S}_{L}^{i} \rightarrow \mathcal{S}_{F}^{j}$, with $x_{F}^{j}=\varphi^{j}\left(\mathbf{x}_{L}\right), j \in \mathcal{F}_{F}$, let $\varphi\left(x_{L}^{i}, \mathbf{x}_{L}^{-i}\right)=\left(\varphi^{1}\left(x_{L}^{i}, \mathbf{x}_{L}^{-i}\right), \ldots, \varphi^{n_{F}}\left(x_{L}^{i}, \mathbf{x}_{L}^{-i}\right)\right)$. The program of leader $i$ consists in maximizing her profit $\pi^{i}\left(x_{L}^{i}, \mathbf{x}_{L}^{-i}, \boldsymbol{\varphi}\left(x_{L}^{i}, \mathbf{x}_{L}^{-i}\right)\right)$ subject to $x_{L}^{i} \in\left[0, X^{*}\right]$, a nonempty and compact (convex) set. Moreover, from Lemma 2 , the effective demand $p\left(x_{L}^{i}+\sum_{-i \neq i} x_{L}^{-i}+\sum_{j} \varphi^{j}\left(x_{L}^{i}, \mathbf{x}_{L}^{-i}\right)\right)$ is continuous as each $\varphi^{j}\left(x_{L}^{i}, \mathbf{x}_{L}^{-i}\right), j \in \mathcal{F}_{F}$, is continuous. Then $\pi^{i}($.$) is a continuous function from \prod_{i} \mathcal{S}_{L}^{i}$ to $\mathcal{S}_{L}^{i}$. Then, the set $\arg \max \left\{\pi^{i}\left(x_{L}^{i}, \boldsymbol{\varphi}\left(x_{L}^{i}, \mathbf{x}_{L}^{-i}\right)\right): x_{L}^{i} \in\left[0, X^{*}\right]\right\}$ is nonempty, so there exists $\psi^{i}: \prod_{-i \neq i} \mathcal{S}_{L}^{-i} \rightarrow \mathcal{S}_{L}^{i}$, with $\psi^{i}\left(\mathbf{x}_{L}^{-i}\right), i \in \mathcal{F}_{L}$. We now characterize leader $i$ 's optimal behavior. Let $\mathcal{L}$ be the Lagrangian and $\lambda^{i}$ and $\mu^{i}$ the Lagrange multipliers. The problem of leader $i \in \mathcal{F}_{L}$ may be written:

$$
\max \mathcal{L}\left(x_{L}^{i}, \mathbf{x}_{L}^{-i}, \lambda^{i}, \mu^{i}\right):=\pi_{L}^{i}\left(x_{L}^{i}, \mathbf{x}_{L}^{-i}, \boldsymbol{\varphi}\left(x_{L}^{i}, \mathbf{x}_{L}^{-i}\right)\right)+\lambda^{i} x_{L}^{i}+\mu^{i}\left(X^{*}-x_{L}^{i}\right) .
$$

The Kuhn-Tucker conditions may be written:

$$
\begin{aligned}
\frac{\partial \mathcal{L}}{\partial x_{L}^{i}} & =\frac{\partial \pi_{L}^{i}}{\partial x_{L}^{i}}+\lambda^{i}-\mu^{i}=0, i \in \mathcal{F}_{L} \\
\lambda^{i} & \geqslant 0, x_{L}^{i} \geqslant 0, \text { with } \lambda^{i} x_{L}^{i}=0 \\
\left(X^{*}-x_{L}^{i}\right) & \geqslant 0, \mu^{i} \geqslant 0, \text { with } \mu^{i}\left(X^{*}-x_{L}^{i}\right)=0,
\end{aligned}
$$

where:

$$
\frac{\partial \pi^{i}}{\partial x_{L}^{i}}=p(X)+\left(1+\nu^{i}\right) x_{L}^{i} \frac{d p(X)}{d X}-\frac{d c^{i}\left(x_{L}^{i}\right)}{d x_{L}^{i}}, i \in \mathcal{F}_{L}
$$


The term $\nu^{i}=\frac{\partial \sum_{j} \varphi^{j}\left(\mathbf{x}_{L}\right)}{\partial x_{L}^{i}}$, with $\nu^{i} \in[-1,0]$, represents the reaction of all followers to leader $i$ 's strategy, i.e., the slope of the aggregate best response to $i, i \in \mathcal{F}_{L}$. By construction $\nu^{i}=\nu^{-i}=\nu$ for all $i,-i \in \mathcal{F}_{L}$. From (1a) we may have either $\psi^{i}\left(\mathbf{x}_{L}^{-i}\right)=0$ or $\psi^{i}\left(\mathbf{x}_{L}^{-i}\right)>0$. Therefore, if $x_{L}^{i}>0$, then $\lambda^{i}=0$, where $x_{L}^{i}$ is the solution to $p(X)+(1+\nu) x_{L}^{i} \frac{d p(X)}{d X}-\frac{d c^{i}\left(x_{L}^{i}\right)}{d x_{L}^{i}}=\mu^{i}$, which then yields $\psi^{i}\left(\mathbf{x}_{L}^{-i}\right)>0$. And, if $\mu^{i}>0$, then $\psi^{i}\left(\mathbf{x}_{L}^{-i}\right)=X^{*}$, since $\mathbf{x}_{L}^{-i}=\mathbf{0}$ and $\mathbf{x}_{F}=\mathbf{0}$, while if $\mu^{i}=0$, then $\psi^{i}\left(\mathbf{x}_{L}^{-i}\right) \in\left(0, X^{*}\right]$. Otherwise, if $x_{L}^{i}=0$, then $p(X)-\frac{d c^{i}\left(x_{L}^{i}\right)}{d x_{L}^{i}}=0$ and $\mu^{i}=0$. Then $\psi^{i}\left(\mathbf{x}_{L}^{-i}\right) \geqslant 0$ whenever $x_{L}^{i} \in\left[0, X^{*}\right]$.

The mapping $\psi^{i}\left(\mathbf{x}_{L}^{-i}\right)$ is a continuous function on $\prod_{-i \neq i} \mathcal{S}_{L}^{-i}$. We first show it is point valued. Differentiating $\frac{\partial \pi_{L}^{i}}{\partial x_{L}^{i}}$ with respect to $x_{L}^{i}$ leads to:

$$
\frac{\partial^{2} \pi^{i}}{\left(\partial x_{L}^{i}\right)^{2}}=(1+\nu)^{2} x_{L}^{i} \frac{d^{2} p(X)}{(d X)^{2}}+2(1+\nu) \frac{d p(X)}{d X}-\frac{d^{2} c_{L}^{i}\left(x_{L}^{i}\right)}{\left(d x_{L}^{i}\right)^{2}} .
$$

We now check that $\frac{\partial^{2} \pi_{L}^{i}}{\left(\partial x_{L}^{i}\right)^{2}}<0$. The expression given by (9) may be written $\frac{\partial^{2} \pi_{L}^{i}}{\left(\partial x_{L}^{i}\right)^{2}}=$ $(1+\nu)^{2}\left(x_{L}^{i} \frac{d^{2} p(X)}{(d X)^{2}}+\frac{d p(X)}{d X}\right)+\left(1-\nu^{2}\right) \frac{d p(X)}{d X}-\frac{d^{2} c_{L}^{i}\left(x_{L}^{i}\right)}{\left(d x_{L}^{i}\right)^{2}}$ by adding $(1+\nu)^{2} \frac{d p(X)}{d X}$ and substracting $\nu(1+\nu) \frac{d p(X)}{d X}$. As the first term is non positive, and the second term is strictly negative under $(2 \mathrm{~b})$, and since $\nu \in[-1,0]$, then we have $\frac{\partial^{2} \pi_{L}^{i}}{\left(\partial x_{L}^{i}\right)^{2}}<0$. Then, the solution to (6), that is $\psi^{i}\left(\mathbf{x}_{L}^{-i}\right), i \in \mathcal{F}_{L}$, is point valued. In addition, $\psi^{i}\left(\mathbf{x}_{L}^{-i}\right), i \in \mathcal{F}_{L}$, is continuous at all $\mathbf{x}_{L}^{-i} \in \prod_{-i} \mathcal{S}^{-i}$, and then on $\prod_{-i} \mathcal{S}^{-i}$.

Remark 2. When $\psi^{i}\left(\mathbf{x}_{L}^{-i}\right) \in\left(0, X^{*}\right](7)$ may be written $L^{i}=-\frac{1+\nu}{\epsilon} \frac{x_{L}^{i}}{X}$, where $L^{i} \equiv \frac{p(X)-\frac{d c_{L}^{i}\left(x_{L}^{i}\right)}{d x_{L}^{i}}}{p(X)}$ is the Lerner index, $\epsilon$ the price elasticity of demand, and $\frac{x_{L}^{i}}{X}$ the market share of leader $i$. In addition, $p(X)=\frac{1}{1-\kappa^{i}} \frac{d c_{L}^{i}\left(x_{L}^{i}\right)}{d x_{L}^{i}}$, where $\frac{1}{1-\kappa^{i}}>1$ is her markup, with $\kappa^{i}=1-\frac{1+\nu}{\epsilon} \frac{x_{L}^{i}}{X}, \kappa^{i} \in[0,1)$. When $\kappa^{i}=0$ (resp. $\left.\kappa^{i}>0\right)$ she behaves as a price taker (resp. an oligopolist). As $\kappa^{i} \in[0,1)$, we have $\nu \geqslant-1$.

Remark 3. Let us notice that $k=(1+\nu)$ in (1c), so using (8), we have:

$$
\frac{\partial^{2} \pi^{i}}{\partial x_{L}^{i} \partial x_{L}^{-i}}=(1+\nu)\left(\frac{d p(X)}{d X}+(1+\nu) x_{L}^{i} \frac{d^{2} p(X)}{(d X)^{2}}\right) \leqslant 0, i \in \mathcal{F}_{L} .
$$

The expression given in (10) echoes Remark 1.

Proposition 4. Let $\boldsymbol{\Psi}: \prod_{i \in \mathcal{F}_{L}} \mathcal{S}^{i} \rightarrow \prod_{i \in \mathcal{F}_{L}} \mathcal{S}^{i}$, with $\boldsymbol{\Psi}=\left(\psi^{1}, \ldots, \psi^{i}, \ldots, \psi^{n_{L}}\right)$. Consider $\mathcal{J}_{\Psi}\left(\overline{\mathbf{x}}_{L}\right)=\left[\frac{\partial\left(\psi^{1}, \ldots, \psi^{i}, \ldots, \psi^{n_{L}}\right)}{\partial\left(x_{L}^{1}, \ldots, x_{L}^{i}, \ldots, x_{L}^{n_{L}}\right)}\right]$. Then, $-\mathcal{I}_{\left(n_{L}, n_{L}\right)}<<\mathcal{J}_{\Psi}\left(\overline{\mathbf{x}}_{L}\right) \leq \mathbf{0}_{\left(n_{L}, n_{L}\right)}$.

Proof. The Jacobian matrix $J_{\Psi}\left(\bar{x}_{L}\right)$ is bounded. Differentiating partially the identity $\frac{\partial \pi_{L}^{i}}{\partial x_{L}^{i}}\left(\psi^{j}\left(\mathbf{x}_{L}^{-i}\right), \mathbf{x}_{L}^{-i}\right) \equiv 0$ with respect to $x_{L}^{-i}$, and using (10), we get $\frac{\partial \psi^{i}}{\partial x_{L}^{-i}}=$ $-\frac{\frac{\partial^{2} \pi_{L}^{i}}{\partial x_{L}^{i} \partial x_{L}^{-i}}}{\frac{\partial^{2} \pi_{L}^{i}}{\partial\left(x_{L}^{i}\right)^{2}}}=-\frac{k \frac{d p(X)}{d X}+k^{2} x_{L}^{i} \frac{d^{2} p(X)}{(d X)^{2}}}{2 k \frac{d p(X)}{d X}+k^{2} x_{L}^{i} \frac{d^{2} p(X)}{(d X)^{2}}-\frac{d^{2} c_{L}^{i}\left(x_{L}^{i}\right)}{\left(d x_{L}^{i}\right)^{2}}}$. From (1b) and (2b), and as for each $i \in \mathcal{F}_{L}, \nu \in[-1,0]$, we deduce $\frac{\partial \phi^{j}}{\partial x_{F}^{-j}} \in(-1,0]$, for all $-i \neq i,-i, i \in \mathcal{F}_{L} . \quad$ । 


\section{SE: EXISTENCE AND UNIQUENESS}

We now turn to the existence and uniqueness of a SE. Before we provide a definition of a SE for the game $\boldsymbol{\Gamma}=\left\{\mathcal{F},\left(\mathcal{S}_{L}^{i}, \pi_{L}^{i}\right),\left(\mathcal{S}_{F}^{j}, \pi_{F}^{j}\right)\right\}_{i \in \mathcal{F}_{L}}^{j \in \mathcal{F}_{F}}$.

Definition 4. (SE) A Stackelberg equilibrium of $\boldsymbol{\Gamma}$ is given by a strategy profile $\left(\tilde{\mathbf{x}}_{L}, \tilde{\mathbf{x}}_{F}\right) \in \prod_{i \in \mathcal{F}_{L}} \mathcal{S}_{L}^{i} \times \prod_{j \in \mathcal{F}_{F}} \mathcal{S}_{F}^{j}$ such that:

i. $\pi_{F}^{j}\left(\tilde{x}_{F}^{j}, \tilde{\mathbf{x}}_{F}^{-j}, \tilde{\mathbf{x}}_{L}\right) \geqslant \pi_{F}^{j}\left(x_{F}^{i}, \tilde{\mathbf{x}}_{F}^{-j}, \tilde{\mathbf{x}}_{L}\right), \forall x_{F}^{j} \in \mathcal{S}^{j}, j \in \mathcal{F}_{F}$

ii. $\pi_{L}^{i}\left(\tilde{x}_{L}^{i}, \tilde{\mathbf{x}}_{L}^{-i}, \boldsymbol{\varphi}\left(\tilde{x}_{L}^{i}, \tilde{\mathbf{x}}_{L}^{-i}\right)\right) \geqslant \pi_{L}^{i}\left(x_{L}^{i}, \tilde{\mathbf{x}}_{L}^{-i}, \boldsymbol{\varphi}\left(x_{L}^{i}, \tilde{\mathbf{x}}_{L}^{-i}\right)\right), \forall \varphi\left(\mathbf{x}_{L}\right) \in \prod_{j \in \mathcal{F}_{F}} \mathcal{S}_{F}^{j}$, $\forall \mathbf{x}_{L}^{-i} \in \prod_{-i \in \mathcal{F}_{L}} \mathcal{S}_{F}^{-i}$ and $\forall x_{L}^{i} \in \mathcal{S}_{L}^{i}, i \in \mathcal{F}_{L}$.

A SE is a noncooperative oligopoly equilibrium of a two-step game of perfect information such that, on the one hand, the market clears, and on the other hand, in each step of the game, no firm has an incentive to unilaterally deviate from its choice. Therefore, we must first consider the mutual consistency of the optimal behavior. Then, we turn to the study of uniqueness.

\subsection{Existence}

The hierarchical model of Sections 2 and 3 consists of two Cournot competitions encompassed by a Stackelberg competition. Indeed, the $n_{L}$ leaders play a twostep game with the $n_{F}$ followers, but the leaders (the followers) play together a simultaneous move game. Stackelberg competition is thus described by a two-step game which embodies two simultaneous move games. Therefore, this hierarchical game $\boldsymbol{\Gamma}$ displays two partial games, namely $\boldsymbol{\Gamma}_{F}$ and $\boldsymbol{\Gamma}_{L}$. The equilibrium of the entire game $\boldsymbol{\Gamma}$ is a pure strategy subgame perfect Nash equilibrium (SPNE), while the equilibria in each partial game are Nash equilibria. Therefore, we must show that there exists a pure strategy SPNE of $\boldsymbol{\Gamma}$. But such a SPNE exists for the entire game if it is a Nash equilibrium of each subgame of $\boldsymbol{\Gamma}$ (Selten (1975)). In addition, we must check that the fixed point is an active equilibrium, i.e., the StackelbergNash equilibrium strategy profile contains strictly positive components. Remark 4 outlines this point.

Remark 4. (Trivial equilibrium). Let us notice that the existence of interior solutions to programs (3) and (6), whose solutions may be well defined optimal decisions, does not ensure that the SE is not a trivial equilibrium. A trivial equilibrium is given by a strategy profile $\left(\tilde{\mathbf{x}}_{L}, \tilde{\mathbf{x}}_{F}\right) \in \prod_{i} \mathcal{S}_{L}^{i} \times \prod_{j} \mathcal{S}_{F}^{j}$ such that $\left(\tilde{\mathbf{x}}_{L}, \tilde{\mathbf{x}}_{F}\right)=(\mathbf{0}, \mathbf{0})$.

For instance, assume $\left|\mathcal{F}_{F}\right|=\left|\mathcal{F}_{L}\right|=1$, with $p(X)=\frac{1}{X}$, and $c_{F}\left(x_{F}\right)=\frac{1}{2} x_{F}$ and $c_{L}\left(x_{L}\right)=0$. Then, the follower's best response is well defined and given by $\phi\left(x_{L}\right)=-x_{L}+\sqrt{2 x_{L}}$, with $\phi\left(x_{L}\right) \geqslant 0$. But the equilibrium strategy profile is $\left(\tilde{x}_{L}, \tilde{x}_{F}\right)=(0,0)$, the trivial solution (note the profit functions are strictly concave).

We are now able to state the following Theorem.

Theorem 1. (Existence). Consider the game $\boldsymbol{\Gamma}=\left\{\mathcal{F},\left(\mathcal{S}_{L}^{i}, \pi_{L}^{i}\right),\left(\mathcal{S}_{F}^{j}, \pi_{F}^{j}\right)\right\}_{i \in \mathcal{F}_{L}}^{j \in \mathcal{F}_{F}}$, and let Assumptions 1 and 2 be satisfied. Then, there exists a Stackelberg equilibrium which is an active equilibrium. 
Proof. The strategy of the proof is as follows. First, we show there exists a strategy profile $\left(\tilde{\mathbf{x}}_{L}, \tilde{\mathbf{x}}_{F}\right) \in \prod_{i} \mathcal{S}_{L}^{i} \times \prod_{j} \mathcal{S}_{F}^{j}$ such that the leaders and followers strategic optimal plans determined in Section 3 are mutually consistent. Second, we show that there may exist the strategy profile $\left(\tilde{\mathbf{x}}_{L}, \tilde{\mathbf{x}}_{F}\right)$ is such that $\left(\tilde{\mathbf{x}}_{L}, \tilde{\mathbf{x}}_{F}\right)>>(\mathbf{0}, \mathbf{0})$.

1. Consistency of optimal plans. The game $\boldsymbol{\Gamma}$ is a two-step game which embodies two simultaneous move games. Let $\boldsymbol{\Gamma}_{L}$ and $\boldsymbol{\Gamma}_{F}$ be the simultaneous move partial games between leaders and between followers respectively. Therefore, we must show that the set of optimal decision functions for each partial game has a fixed point, which also constitutes a Nash equilibrium for the entire game.

We first show there is a fixed point to the system of leader's optimal decision mappings. From Lemmas 1-3, we can define $\psi^{i}: \prod_{-i} \mathcal{S}_{L}^{-i} \rightarrow \mathcal{S}_{L}^{i}$, with $\psi^{i}\left(\mathbf{x}_{L}^{-i}\right), i \in \mathcal{F}_{L}$. Now define the function $\boldsymbol{\Lambda}_{L}: \prod_{i} \mathcal{S}_{L}^{i} \rightarrow \prod_{i} \mathcal{S}_{L}^{i}$, with $\boldsymbol{\Lambda}_{L}\left(\mathbf{x}_{L}\right)=\times_{i=1}^{n_{L}} \psi^{i}$. The function $\boldsymbol{\Lambda}\left(\mathbf{x}_{L}\right)$ is continuous (as each $\psi^{i}$ is continuous) in $\mathbf{x}_{L}$ on $\prod_{i} \mathcal{S}_{L}^{i}$, a compact and convex subset of Euclidean space (as the product of compact and convex sets $\mathcal{S}_{L}^{i}$, $\left.i \in \mathcal{F}_{L}\right)$. Then, by the Brouwer Fixed Point Theorem, the function $\boldsymbol{\Lambda}\left(\mathbf{x}_{L}\right)$ admits a fixed point $\tilde{\mathbf{x}}_{L} \in \prod_{i} \mathcal{S}_{L}^{i}$, with components $\tilde{x}_{L}^{i}$, with $\tilde{x}_{L}^{i} \in \mathcal{S}_{L}^{i}, i \in \mathcal{F}_{L}$. This fixed point constitutes a pure strategy Nash equilibrium of $\boldsymbol{\Gamma}_{L}$.

We now show there is a fixed point to the system of followers optimal decision mappings. Using Lemma 1, we can define $\boldsymbol{\Lambda}_{F}: \prod_{j} \mathcal{S}_{F}^{j} \times \prod_{i} \mathcal{S}_{L}^{i} \rightarrow \prod_{j} \mathcal{S}_{F}^{j} \times \prod_{i} \mathcal{S}_{L}^{i}$, with $\boldsymbol{\Lambda}_{F}\left(\mathbf{x}_{F}, \mathbf{x}_{L}\right)=\times_{j=1}^{n_{F}} \phi^{j}$. Given $\tilde{\mathbf{x}}_{L} \in \prod_{i} \mathcal{S}_{L}^{i}$, we have $\boldsymbol{\Lambda}_{F}\left(\mathbf{x}_{F}, \tilde{\mathbf{x}}_{L}\right)=\times_{j=1}^{n_{F}} \phi^{j}$. A similar argument as the one made for the leaders shows that the function $\boldsymbol{\Lambda}_{F}\left(\mathbf{x}_{F}, \tilde{\mathbf{x}}_{L}\right)$ admits a fixed point $\tilde{\mathbf{x}}_{F} \in \prod_{j} \mathcal{S}_{F}^{j}$, with components $\tilde{x}_{F}^{j} \in \mathcal{S}_{F}^{j}, j \in \mathcal{F}_{F}$. This fixed point is a pure strategy Nash equilibrium of $\boldsymbol{\Gamma}_{F}$. Now we must show that there is a Nash equilibrium for the entire game $\boldsymbol{\Gamma}$. From Lemma 2, we can define $\varphi^{j}: \prod_{i} \mathcal{S}_{L}^{i} \rightarrow \mathcal{S}_{F}^{j}$, with $x_{F}^{j}=\varphi^{j}\left(\mathbf{x}_{L}\right), i \in \mathcal{F}_{L}$. Consider the vector function of best responses $\mathbf{x}_{F}=\boldsymbol{\varphi}\left(\mathbf{x}_{L}\right)$, with $\mathbf{x}_{F} \in \prod_{j} \mathcal{S}_{L}^{j}$. If $\tilde{\mathbf{x}}_{L} \in \prod_{i} \mathcal{S}_{L}^{i}$ is a pure strategy Nash equilibrium of $\boldsymbol{\Gamma}_{L}$, then, by using Lemma 2, we have $\tilde{\mathbf{x}}_{F}=\boldsymbol{\varphi}\left(\tilde{\mathbf{x}}_{L}\right) \in \prod_{j} \mathcal{S}_{F}^{j}$. Then, to $\tilde{\mathbf{x}}_{L} \in \prod_{i} \mathcal{S}_{L}^{i}$ corresponds a strategy profile $\tilde{\mathbf{x}}_{F} \in \prod_{j} \mathcal{S}_{F}^{j}$, which constitutes a pure strategy Nash equilibrium of $\boldsymbol{\Gamma}_{F}$. We conclude there exists a strategy profile $\left(\tilde{\mathbf{x}}_{L}, \tilde{\mathbf{x}}_{F}\right) \geq(\mathbf{0}, \mathbf{0})$, with $\left(\tilde{\mathbf{x}}_{L}, \tilde{\mathbf{x}}_{F}\right) \in \prod_{i} \mathcal{S}_{L}^{i} \times \prod_{j} \mathcal{S}_{F}^{j}$, which constitutes a SPNE of $\boldsymbol{\Gamma}$. Then, from $\tilde{X}$ we deduce $\tilde{p}(\tilde{X})$, with $\tilde{X} \in\left[0, X^{*}\right]$. The payoffs $\tilde{\pi}^{i}\left(\tilde{\mathbf{x}}_{L}, \tilde{\mathbf{x}}_{F}\right), i \in \mathcal{F}_{L}$, and $\tilde{\pi}^{j}\left(\tilde{\mathbf{x}}_{L}, \tilde{\mathbf{x}}_{F}\right), j \in \mathcal{F}_{F}$, follow from (1) and (2).

2. Equilibrium strategy profiles. The equilibrium strategy profile may be the trivial one, in which case $\left(\tilde{\mathbf{x}}_{L}, \tilde{\mathbf{x}}_{F}\right)=(\mathbf{0}, \mathbf{0})$, or interior, i.e., $\left(\tilde{\mathbf{x}}_{L}, \tilde{\mathbf{x}}_{F}\right)>>(\mathbf{0}, \mathbf{0})$, with $\left(\tilde{\mathbf{x}}_{L}, \tilde{\mathbf{x}}_{F}\right) \in \prod_{i} \mathcal{S}^{i} \times \prod_{j} \mathcal{S}^{j}$. So, we must now show that for any leader $i \in \mathcal{F}_{L}$ (resp. follower $j \in \mathcal{F}_{F}$ ), there may exist lower and upper bounds $\check{x}_{L}^{i}$ and $\bar{x}_{L}^{i}$ (resp. $\check{x}_{F}^{j}$ and $\bar{x}_{F}^{j}$ ) on equilibrium supplies such that $0<\check{x}_{L}^{i} \leqslant \tilde{x}_{L}^{i} \leqslant \bar{x}_{L}^{i}<\infty, i \in \mathcal{F}_{L}$ (resp. $0<\check{x}_{F}^{j} \leqslant \tilde{x}_{F}^{j} \leqslant \bar{x}_{F}^{j}<\infty, j \in \mathcal{F}_{F}$ ).

Existence of upper bound: $\tilde{x}_{L}^{i} \leqslant \bar{x}_{L}^{i}<\infty, i \in \mathcal{F}_{L}$. There are two cases to deal with: either the inverse demand function intersects the axes or it does not. 
Assume first the inverse demand function $p(X)$ intersects the quantity axis. In such a case, $p(X)$ may be either (strictly) concave or (strictly) convex. Therefore, there is $\bar{X}<\infty$ such that $p(X)>0$ for $X<\bar{X}$, and $p(\bar{X})=0$ for $X \geqslant \bar{X}$. Therefore, there is some finite quantity demanded when the commodity is a free good. Select one leader $i \in \mathcal{F}_{L}$. Consider $\bar{x}_{L}^{i}=\max \left(\theta_{L}^{1} \bar{X}, \ldots, \theta_{L}^{n_{L}} \bar{X} ; \theta_{F}^{1} \bar{X}, \ldots, \theta_{F}^{n_{F}} \bar{X}\right)$, with $\theta_{L}^{i}, \theta_{F}^{j} \in$ $[0,1]$, for each $i \in \mathcal{F}_{L}$ and each $j \in \mathcal{F}_{F}$, where $\sum_{i}^{n_{L}} \theta_{L}^{i}+\sum_{j}^{n_{F}} \theta_{F}^{j}=1$. As $\bar{X}<\infty$, then $\bar{x}_{L}^{i}<\infty$. Then, $\bar{x}_{L}^{i}<\infty, i \in \mathcal{F}_{L}$, and $\bar{x}_{F}^{j}<\infty, j \in \mathcal{F}_{F}$. Assume now $\lim _{X \rightarrow 0} p(X)=\infty$ and $\lim _{X \rightarrow X^{*}} p(X)=\infty$. Consider there is some $i \in \mathcal{F}_{L}$ for which $\tilde{x}_{L}^{i}=\infty$. But, from (2c), we know that $\frac{d c_{L}^{i}}{d x_{L}^{i}}\left(\tilde{x}_{L}^{i}\right)>p\left(\tilde{x}_{L}^{i}\right)$, so we must have $\tilde{x}_{L}^{i}<\infty$. The same argument holds for all $i \in \mathcal{F}_{L}$, and for all $j \in \mathcal{F}_{F}$.

Existence of lower bound: $0<\check{x}_{L}^{i} \leqslant \tilde{x}_{L}^{i}, i \in \mathcal{F}_{L}$. The proof holds either when $\lim _{X \rightarrow 0} p(X)=\bar{p}$, with $0<p<\infty$, or when $\lim _{X \rightarrow 0} p(X)=\infty$. Assume $\tilde{x}_{L}^{i}=0$, with $c_{L}^{i}\left(\tilde{x}_{L}^{i}\right)=\min \left\{c_{L}^{1}\left(\tilde{x}_{L}^{1}\right), \ldots, c_{L}^{n_{L}}\left(\tilde{x}_{L}^{n_{L}}\right) ; c_{F}^{1}\left(\tilde{x}_{F}^{1}\right), \ldots, c_{F}^{n_{F}}\left(\tilde{x}_{F}^{n_{F}}\right)\right\}$. Let $\tilde{x}_{L}^{-i}>0$, for all $-i \neq i,-i \in \mathcal{F}_{L}$, and $\tilde{x}_{F}^{j}>0$, for all $j \in \mathcal{F}_{F}$. As $\tilde{x}_{L}^{i}=0$, from (9), we deduce $\lambda^{i} \geqslant 0$, so the first-order condition is given by $p(\tilde{X})+\lambda^{i}=c_{L}^{i}\left(\tilde{x}_{L}^{i}\right)$, which implies $p(\tilde{X}) \leqslant c_{L}^{i}(0)$. Therefore, the effective demand which addresses to any leader $-i \neq i$ is given by $p\left(\tilde{X}_{L}^{-i}+\tilde{X}_{F}\left(\tilde{X}_{L}^{-i}\right)\right)$. As from (1b), we have $\frac{d p(X)}{d X}<0$, then assuming $\tilde{x}_{L}^{-i}>0$ leads to $p\left(\tilde{X}_{L}^{-i}+\tilde{X}_{F}\left(\tilde{X}_{L}^{-i}\right)\right)+k x_{L}^{-i} \frac{d p}{d X}\left(\tilde{X}_{L}^{-i}+\tilde{X}_{F}\left(\tilde{X}_{L}^{-i}\right)\right)-\frac{d c_{L}^{-i}}{d x_{L}^{-i}}\left(\tilde{x}_{L}^{-i}\right)<0$, $-i \in \mathcal{F}_{L}$, since $\frac{d c_{L}^{-i}}{d x_{L}^{-i}}\left(\tilde{x}_{L}^{-i}\right) \geqslant \frac{d c_{L}^{i}}{d x_{L}^{i}}\left(\tilde{x}_{L}^{i}\right)$. Then, we deduce $\tilde{x}_{L}^{-i}=0$ for all $-i \in \mathcal{F}_{L}$. But then, for each $-i \in \mathcal{F}_{L}, p(0) \leqslant \frac{d c_{L}^{-i}}{d x_{L}^{-i}}(0)$, a contradiction, as (2c) must hold. In consequence, if the residual demand which addresses to any follower $j \in \mathcal{F}_{F}$ is $p\left(0+\tilde{X}_{F}\right)$, then, from (4) and Lemma 2, we deduce $p\left(\tilde{X}_{F}\right)+x_{F}^{j} \frac{d p}{d X}\left(\tilde{X}_{F}\right)-\frac{d c_{F}^{j}}{d x_{F}^{j}}\left(\tilde{x}_{F}^{j}\right)<$ $0, j \in \mathcal{F}_{F}$, since $\frac{d c_{F}^{j}}{d x_{F}^{j}}\left(\tilde{x}_{F}^{j}\right) \geqslant \frac{d c_{L}^{i}}{d x_{L}^{i}}\left(\tilde{x}_{L}^{i}\right)$. But then, for each $j \in \mathcal{F}_{F}, p(0) \leqslant \frac{d c_{F}^{j}}{d x_{F}^{j}}(0)$, so $\tilde{x}_{F}^{j}=0$, for all $j \in \mathcal{F}_{F}$. A contradiction. Then, we conclude that there must exist some firm $i \in \mathcal{F}_{L}$ for which $\breve{x}_{L}^{i}>0$, and some firm $j \in \mathcal{F}_{F}$ for which $\breve{x}_{F}^{j}>0$.

Thus, the second part of the proof of Theorem 2 reveals that there is always a more profitable strategic supply than the inactive one.

\subsection{Uniqueness}

Uniqueness is not guaranteed since we should have a configuration in which the best responses are uniquelly determined but there could exist multiple equilibrium leaders' strategy profiles (see Example 4 in Section 5).

Nevertheless, we are able to state the following Theorem.

Theorem 2. (Uniqueness). Let Assumptions 1 and 2 be satisfied. Then, if there exists an active Stackelberg equilibrium, then it is unique.

Proof. The strategy of the proof is as follows. We show that if the partial game $\boldsymbol{\Gamma}_{L}$ has a unique active Nash equilibrium, namely $\tilde{\mathbf{x}}_{L} \in \prod_{i} \mathcal{S}_{L}^{i} \backslash\{\mathbf{0}\}$, then the equilibrium $\tilde{\mathbf{x}}_{F} \in \prod_{j} \mathcal{S}_{F}^{j}$ in the partial game $\boldsymbol{\Gamma}_{F}$ is unique as the function $\varphi$ defined as $\boldsymbol{\varphi}: \prod_{i} \mathcal{S}_{L}^{i} \supset \mathcal{U}_{\left(\overline{\mathbf{x}}_{L}\right)} \rightarrow \prod_{j} \mathcal{S}_{F}^{j}$, with $\mathbf{x}_{F}=\boldsymbol{\varphi}\left(\mathbf{x}_{L}\right)$, is one to one. Then, uniqueness of the SNPE for $\boldsymbol{\Gamma}$ (and then uniqueness of SE) is obtained through Lemma 2. 
To this end, consider the gradient vector $\boldsymbol{\Pi}_{L}=\left(\frac{\partial \pi_{L}^{1}}{\partial x_{L}^{1}}, \ldots, \frac{\partial \pi_{L}^{i}}{\partial x_{L}^{i}}, \ldots, \frac{\partial \pi_{L}^{n_{L}}}{\partial x_{L} L}\right)$. Let an active equilibrium strategy profile $\left(\tilde{\mathbf{x}}_{L}, \tilde{\mathbf{x}}_{F}\right) \in \prod_{i} \mathcal{S}_{L}^{i} \times \prod_{j} \mathcal{S}_{F}^{j}$. Then, consider the determinant of the Jacobian matrix of $-\boldsymbol{\Pi}_{L}$ at $\left(\tilde{\mathbf{x}}_{L}, \tilde{\mathbf{x}}_{F}\right)$, which we denote by $\left|J_{-\boldsymbol{\Pi}_{L}}\left(\tilde{\mathbf{x}}_{L}, \tilde{\mathbf{x}}_{F}\right)\right|$. It is well known that if the determinant at an active Cournot equilibrium is positive, then the equilibrium is unique (see Corollary 2.1 in Kolstad and Mathiesen (1987)). As leaders in the partial game $\boldsymbol{\Gamma}_{L}$ behave as Cournot firms, we show this criterion is satisfied, so the pure strategy Nash equilibrium in the partial game $\boldsymbol{\Gamma}_{L}$ is unique. Then, using Lemma 2, we deduce there is a unique pure strategy Nash equilibrium of $\boldsymbol{\Gamma}_{F}$.

Select one strategy profile $\left(\tilde{\mathbf{x}}_{L}, \tilde{\mathbf{x}}_{F}\right) \in \prod_{i} \mathcal{S}_{L}^{i} \times \prod_{j} \mathcal{S}_{F}^{j}$, with $\tilde{\mathbf{x}}_{L}>>\mathbf{0}$. We have to show that $\left|J_{-\Pi_{L}}\left(\tilde{\mathbf{x}}_{L}, \tilde{\mathbf{x}}_{F}\right)\right|>0$. Given $\left(\tilde{\mathbf{x}}_{L}, \tilde{\mathbf{x}}_{F}\right)$, consider the Jacobian matrix $J_{-\Pi_{L}}\left(\left(\tilde{\mathbf{x}}_{L}, \tilde{\mathbf{x}}_{F}\right)=-\left(\frac{\partial^{2} \pi_{L}^{i}}{\partial x_{L}^{i} \partial x_{L}^{-i}}\right), i,-i \in \mathcal{F}_{L}\right.$, with $\frac{\partial \pi_{L}^{i}}{\partial x_{L}^{i}}=p(X)+k x_{L}^{i} \frac{d p(X)}{d X}-\frac{d c_{L}^{i}\left(x_{L}^{i}\right)}{d x_{L}^{i}}$, and where $\frac{\partial^{2} \pi_{L}^{i}}{\left(\partial x_{L}^{i}\right)^{2}}$ and $\frac{\partial^{2} \pi_{L}^{i}}{\partial x_{L}^{i} \partial x_{L}^{-i}}$ are given by (9) and (10) respectively.

If (1a) and (2a) are satisfied, then, by substracting columm 1 to the other columns and expanding by co-factors, we deduce:

$$
\left|J_{-\Pi_{L}}\right|=\left(1-k \sum_{i \in \mathcal{F}_{L}} \frac{\frac{d p(X)}{d X}+k x_{L}^{i} \frac{d^{2} p(X)}{(d X)^{2}}}{\frac{d^{2} c_{L}^{i}\left(x_{L}^{i}\right)}{\left(d x_{L}^{i}\right)^{2}}-k \frac{d p(X)}{d X}}\right) \prod_{i \in \mathcal{F}_{L}}\left(\frac{d^{2} c_{L}^{i}\left(x_{L}^{i}\right)}{\left(d x_{L}^{i}\right)^{2}}-k \frac{d p(X)}{d X}\right) .
$$

Assuming (2b), we deduce:

$$
\operatorname{sign}\left|J_{-\Pi_{L}}\right|=\operatorname{sign}\left(1-k \sum_{i \in \mathcal{F}_{L}} \frac{\frac{d p(X)}{d X}+k x_{L}^{i} \frac{d^{2} p(X)}{(d X)^{2}}}{\frac{d^{2} c_{L}^{i}\left(x_{L}^{i}\right)}{\left(d x_{L}^{i}\right)^{2}}-k \frac{d p(X)}{d X}}\right) .
$$

From (1c) and (2b), we deduce $\mid J_{-\Pi_{L}}\left(\left(\tilde{\mathbf{x}}_{L}, \tilde{\mathbf{x}}_{F}\right) \mid>0\right.$. Then, there exists a unique pure strategy Nash equilibrium in the partial game $\boldsymbol{\Gamma}_{L}$. Now set $\tilde{\mathbf{x}}_{L}>>\mathbf{0}$. From Lemma 2, we deduce $\tilde{\mathbf{x}}_{F}=\boldsymbol{\varphi}\left(\tilde{\mathbf{x}}_{L}\right)$, which constitutes a unique pure strategy Nash equilibrium of $\boldsymbol{\Gamma}_{F}$. Then, we conclude the pure strategy SPNE in $\boldsymbol{\Gamma}$ is unique, which proves uniqueness of the SE.

Assuming symmetry among leaders, the condition for the sign of $\mid J_{-\Pi_{L}}\left(\left(\tilde{\mathbf{x}}_{L}, \tilde{\mathbf{x}}_{F}\right) \mid\right.$ might be rewritten as $\frac{d p(X)}{d X}+k x_{L}^{i} \frac{d^{2} p(X)}{(d X)^{2}}<\frac{1}{k n_{L}}\left(\frac{d^{2} c_{L}^{i}\left(x_{L}^{i}\right)}{\left(d x_{L}^{i}\right)^{2}}-k \frac{d p(X)}{d X}\right)$, which would say that "on average" leaders' marginal revenues could be increased but not too much (see Kolstad and Mathiesen (1987) for the Cournot market).

In addition, we can check that, under $(2 \mathrm{~b})$, we would have had $\frac{d^{2} c_{L}^{i}\left(x_{L}^{i}\right)}{\left(d x_{L}^{i}\right)^{2}}-$ $k \frac{d p(X)}{d X}+n_{L}\left(\frac{d p(X)}{d X}+k x_{L}^{i} \frac{d^{2} p(X)}{(d X)^{2}}\right)=\frac{\partial^{2} \pi_{L}^{1}}{\left(\partial x_{L}^{1}\right)^{2}}+\left(n_{L}-1\right) \frac{\partial^{2} \pi_{L}^{i}}{\partial x_{L}^{i} \partial x_{L}^{-i}}<0$ : the effect on $i$ 's marginal profit of a change in $x_{L}^{i}$ dominates the sum of the cross effects of similar changes of other leaders' supply.

Let us notice that strategic complementarities are not precluded here, but they are not sufficiently strong to compensate strategic substituabilities. Therefore, our result transposes in a Stackelberg framework a result which holds in the Cournot market (see notably Vives (1999)). 


\section{DISCUSSION: SOME EXAMPLES}

In order to discuss Theorems 1 and 2 and to illustrate our consistency criterion (Lemma 2), we provide four examples. We put forward the decreasing marginal revenue assumption which leads to consider the behavior of the price function on the boundary (hypothesis (2a)). We know that this condition may not be written in the same way for the leaders and for the followers (see Remarks 1 and 3). We also put forward the possibility of marginal costs to decrease more than the price function, letting the possibility of increasing returns (hypothesis (2b)). Example 1 outlines the procedure to compute a SE when Assumptions 1 and 2 hold. Example 2 shows that even if Assumption 1 is not satisfied, a unique SE may exist. Example 3 illustrates existence failure, a case for which our criterion is not satisfied. Example 4 provides a case with multiple equilibria. These examples illustrate that our criterion constitutes a sufficient test condition for the existence of best responses. They also put forward that condition (12) is only sufficient for uniqueness of SE. We assume $\left|\mathcal{F}_{F}\right|=\left|\mathcal{F}_{L}\right|=2$. To save notations, we sometimes let $X_{F} \equiv x_{F}^{1}+x_{F}^{2}$ and $X_{L} \equiv x_{L}^{1}+x_{L}^{2}$.

\subsection{Existence and uniqueness with Assumptions 1 and 2}

Let the inverse market demand function be given by:

$$
p(X)=1-X .
$$

The cost functions are $c_{L}^{i}\left(x_{L}^{i}\right)=\ln \left(1+\frac{1}{2} x_{L}^{i}\right), i=1,2, c_{F}^{1}\left(x_{F}^{1}\right)=\frac{1}{2}\left(x_{F}^{1}\right)^{2}$ and $c_{F}^{2}\left(x_{F}^{2}\right)=\frac{1}{2} x_{F}^{2}$. Let $\max \left\{\frac{1 / 2}{1+(1 / 2) x_{L}^{i}}, x_{F}^{1}, \frac{1}{2}\right\}=\frac{1}{2}$. From (13) we get $X^{*}=\frac{1}{2}$, so $x_{L}^{i} \in\left[0, \frac{1}{2}\right], i=1,2$, and $x_{F}^{j} \in\left[0, \frac{1}{2}\right], j=1,2$.

The followers's optimal decisions corresponding to (4) are given by:

$$
\begin{aligned}
& \phi^{1}\left(x_{F}^{2}, \mathbf{x}_{L}\right)=\frac{1}{3}-\frac{1}{3}\left(x_{F}^{2}+X_{L}\right) \\
& \phi^{2}\left(x_{F}^{1}, \mathbf{x}_{L}\right)=\frac{1}{4}-\frac{1}{2}\left(x_{F}^{1}+X_{L}\right) .
\end{aligned}
$$

Using (5), we get $\Phi^{1}\left(\mathbf{x}_{F}, \mathbf{x}_{L}\right) \equiv x_{F}^{1}-\frac{1}{3}+\frac{x_{F}^{2}+X_{L}}{3}$ and $\Phi^{2}\left(\mathbf{x}_{F}, \mathbf{x}_{L}\right) \equiv x_{F}^{2}-\frac{1}{4}+$ $\frac{x_{F}^{1}+X_{L}}{2}$. Implicit differentiation of $\Phi^{j}\left(\varphi^{1}\left(\mathbf{x}_{L}\right), \varphi^{2}\left(\mathbf{x}_{L}\right), \mathbf{x}_{L}\right) \equiv 0, j=1,2$, leads to:

$$
\left[\begin{array}{ll}
1 & \frac{1}{3} \\
\frac{1}{2} & 1
\end{array}\right]\left[\begin{array}{ll}
\frac{\partial x_{F}^{1}}{\partial x_{L}^{1}} & \frac{\partial x_{F}^{1}}{\partial x_{L}^{2}} \\
\frac{\partial x_{F}^{2}}{\partial x_{L}^{1}} & \frac{\partial x_{F}^{2}}{\partial x_{L}^{2}}
\end{array}\right]=-\left[\begin{array}{cc}
\frac{1}{3} & \frac{1}{3} \\
\frac{1}{2} & \frac{1}{2}
\end{array}\right] .
$$

As $\left|\mathcal{J}_{\mathbf{\Phi}_{\mathbf{x}_{F}}}\left(\mathbf{x}_{F}, \mathbf{x}_{L}\right)\right|=\frac{5}{6}>0$, we can determine the best responses:

$$
\begin{aligned}
\varphi^{1}\left(\mathbf{x}_{L}\right) & =\frac{3}{10}-\frac{1}{5} X_{L} \\
\varphi^{2}\left(\mathbf{x}_{L}\right) & =\frac{1}{10}-\frac{2}{5} X_{L} .
\end{aligned}
$$

Let us notice $\frac{\partial \varphi^{1}\left(\mathbf{x}_{L}\right)}{\partial x_{L}^{i}}=-\frac{1}{5}$ and $\frac{\partial \varphi^{2}\left(\mathbf{x}_{L}\right)}{\partial x_{L}^{i}}=-\frac{2}{5}$, which may be obtained by applying Cramer's rule to (16). In addition, we have $\nu=-\frac{3}{5}$. Using (17)-(18), 
leader $i$ faces the effective demand $\left(\frac{3}{5}-\frac{2}{5} x_{L}^{i}-\frac{2}{5} x_{L}^{-i}\right)$, so her program is max $\left(\frac{3}{5}-\frac{2}{5} x_{L}^{i}-\frac{2}{5} x_{L}^{-i}\right) x_{L}^{i}-\ln \left(1+\frac{1}{2} x_{L}^{i}\right)$. We deduce $\left(\tilde{x}_{F}^{1}, \tilde{x}_{F}^{2}\right)=\left(\frac{18-\sqrt{105}}{30}, \frac{21-2 \sqrt{105}}{30}\right)$. The residual demand $p(X)=\frac{15-\sqrt{105}}{6}-X_{F}$ leads to (14)-(15).

In addition, Assumptions 1 and 2 are satisfied for any leader:

$$
\begin{aligned}
\frac{d p(X)}{d X}+(1+\nu) \tilde{x}_{L}^{i} \frac{d^{2} p(X)}{(d X)^{2}} & =-1 \leqslant 0, i=1,2 \\
\frac{d^{2} c^{i}\left(x_{L}^{i}\right)}{\left(d x_{L}^{i}\right)^{2}} & =-\frac{144}{(15+\sqrt{105})^{2}}>-\frac{2}{5}=(1+\nu) \frac{d p(X)}{d X},
\end{aligned}
$$

and for any follower:

$$
\begin{aligned}
\frac{d p(X)}{d X}+\tilde{x}_{F}^{j} \frac{d^{2} p(X)}{(d X)^{2}} & =-1<0, j=1,2 \\
\frac{d^{2} c_{F}^{1}\left(x_{F}^{1}\right)}{\left(d x_{F}^{1}\right)^{2}} & =1>-1=\frac{d p(X)}{d X} \\
\text { and } \frac{d^{2} c_{F}^{2}\left(x_{F}^{2}\right)}{\left(d x_{F}^{2}\right)^{2}} & =0>-1=\frac{d p(X)}{d X} .
\end{aligned}
$$

The marginal revenue of any firm decreases, which illustrates the fact that supplies are strategic substitutes between leaders and /or followers.

Finally, (12) yields sign $\left|J_{-\Pi_{L}}\left(\tilde{\mathbf{x}}_{L}, \tilde{\mathbf{x}}_{F}\right)\right|=\operatorname{sign}\left(1+\frac{4(15+\sqrt{105})^{2}}{2(15+\sqrt{105})^{2}-720}\right)>0$. So the SE is unique. We could also check that $\operatorname{sign}\left|J_{-\Pi_{F}}\left(\tilde{\mathbf{x}}_{L}, \tilde{\mathbf{x}}_{F}\right)\right|=\operatorname{sign}\left(\frac{5}{2}\right)>0$.

\subsection{Existence and uniqueness without Assumption 1}

Let the inverse market demand function be given by:

$$
p(X)=\frac{1}{X^{\beta}}, \beta>2,
$$

where $\lim _{X \rightarrow 0} p(X)=\infty, \lim _{X \rightarrow \infty} p(X)=0, \frac{d p(X)}{d X}=-\frac{\beta}{X^{\beta+1}}$ and $\frac{d^{2} p(X)}{(d X)^{2}}=\frac{\beta(\beta+1)}{X^{\beta+2}}$. Let $c_{L}^{i}\left(x_{L}^{i}\right)=\frac{1}{2} x_{L}^{i}, i=1,2$, and $c_{F}^{j}\left(x_{F}^{j}\right)=0, j=1,2$. Let $\max \left\{\frac{1}{2}, 0\right\}=\frac{1}{2}$, from (21), we take $X^{*}=\frac{1}{2}$, so $x_{L}^{i} \in\left[0, \frac{1}{2}\right], i=1,2$, and $x_{F}^{j} \in\left[0, \frac{1}{2}\right], j=1,2$.

The optimal decisions corresponding to (4) are given by:

$$
\begin{aligned}
& \phi^{1}\left(x_{F}^{2}, \mathbf{x}_{L}\right)=\frac{x_{F}^{2}+X_{L}}{\beta-1} \\
& \phi^{2}\left(x_{F}^{2}, \mathbf{x}_{L}\right)=\frac{x_{F}^{1}+X_{L}}{\beta-1} .
\end{aligned}
$$

Let $\Phi^{1}\left(\mathbf{x}_{F}, \mathbf{x}_{L}\right) \equiv x_{F}^{1}-\frac{x_{F}^{2}+X_{L}}{\beta-1}$ and $\Phi^{2}\left(\mathbf{x}_{F}, \mathbf{x}_{L}\right) \equiv x_{F}^{2}-\frac{x_{F}^{1}+X_{L}}{\beta-1}$. We deduce:

$$
\left|\mathcal{J}_{\mathbf{\Phi}_{\mathbf{x}_{F}}}\left(\mathbf{x}_{F}, \mathbf{x}_{L}\right)\right|=\left|\begin{array}{ll}
1 & -\frac{1}{\beta-1} \\
-\frac{1}{\beta-1} & 1
\end{array}\right|=\frac{\beta(\beta-2)}{(\beta-1)^{2}} \in(0,1) \text { as } \beta>2 .
$$

If $\beta=2$ there is no solution to $\Phi^{j}\left(\mathbf{x}_{L}, \mathbf{x}_{F}\right)=0, j=1,2$. Otherwise, we get $\varphi^{j}\left(\mathbf{x}_{L}\right)=\frac{X_{L}}{\beta-2}, j \in \mathcal{F}_{F}$. Let us notice that $\nu^{1}=\frac{2}{\beta-2}>0$, so leaders and followers 
strategies are strategic complements. Using (22)-(23), leader $i$ faces the effective demand $\left(\frac{\beta-2}{\beta} \frac{1}{x_{L}^{i}+x_{L}^{-i}}\right)$, so she solves $\max \frac{\beta-2}{\beta} \frac{x_{L}^{i}}{x_{L}^{i}+x_{L}^{-i}}-\frac{1}{2} x_{L}^{i}$. We deduce $\tilde{x}_{L}^{i}=\frac{1}{2} \frac{\beta-2}{\beta}$, $i=1,2$. Therefore, $\tilde{x}_{F}^{j}=\frac{1}{\beta}, j=1,2$.

We check that $(1 b)$ neither holds for the leaders:

$$
\begin{aligned}
\frac{d p(X)}{d X}+(1+\nu) \tilde{x}_{L}^{i} \frac{d^{2} p(X)}{(d X)^{2}} & =\frac{\beta(\beta-1)}{2}>0, i=1,2 \\
\frac{d^{2} c_{L}^{i}\left(x_{L}^{i}\right)}{\left(d x_{L}^{i}\right)^{2}} & =0>-\frac{2 \beta}{\beta-2}=(1+\nu) \frac{d p(X)}{d X},
\end{aligned}
$$

nor for the followers:

$$
\begin{aligned}
\frac{d p(X)}{d X}+\tilde{x}_{F}^{j} \frac{d^{2} p(X)}{(d X)^{2}} & =1>0 \\
\frac{d^{2} c_{F}^{j}\left(x_{F}^{j}\right)}{\left(d x_{F}^{j}\right)^{2}} & =0>-1=\frac{d p(X)}{d X}, j=1,2 .
\end{aligned}
$$

Here marginal revenue increases; so existence of a SE is not inconsistent with strategic complementarities.

Finally, we have $\operatorname{sign} \mid J_{-\Pi_{L}}\left(\left(\tilde{\mathbf{x}}_{L}, \tilde{\mathbf{x}}_{F}\right) \mid=\operatorname{sign}\left(1-\frac{\beta(\beta-1)}{2}\right)<0\right.$ : the $\mathrm{SE}$ is unique even if the sufficient condition for uniqueness is not satisfied. In addition, $\operatorname{sign} \mid J_{\Pi_{F}}\left(\left(\tilde{\mathbf{x}}_{L}, \tilde{\mathbf{x}}_{F}\right) \mid=\operatorname{sign}(-1)<0\right.$ : strategic complementarities are here not sufficiently strong to produce multiple equilibria.

\subsection{Existence failure (without Assumption 2)}

The price function is given by (13). Let $c_{L}^{i}\left(x_{L}^{i}\right)=0, i=1,2, c_{F}^{1}\left(x_{F}^{1}\right)=$ $1+\alpha_{F}^{i} x_{F}^{i}-\frac{1}{2}\left(x_{F}^{i}\right)^{2}, j=1,2$, with $\alpha_{F}^{1} \neq \alpha_{F}^{2}$ and $\alpha_{F}^{1}, \alpha_{F}^{2}<\infty$. Therefore costs decreases whenever $x_{F}^{j}>\alpha_{F}^{j}, j=1,2$. Let $\max \left\{0, \alpha_{F}^{1}-x_{F}^{1}, \alpha_{F}^{2}-x_{F}^{2}\right\}=\xi$, where $0<\xi<\infty$, so $x_{L}^{i} \in[0, \xi], i=1,2$, and $x_{F}^{j} \in[0, \xi], j=1,2$.

Followers' optimal decisions are given by:

$$
\begin{gathered}
\phi^{1}\left(x_{F}^{2}, \mathbf{x}_{L}\right)=1-\alpha_{F}^{1}-x_{F}^{2}-X_{L} \\
\phi^{2}\left(x_{F}^{1}, \mathbf{x}_{L}\right)=1-\alpha_{F}^{2}-x_{F}^{1}-X_{L} .
\end{gathered}
$$

Using (5), we get $\Phi^{1}\left(\mathbf{x}_{F}, \mathbf{x}_{L}\right) \equiv x_{F}^{1}-1+\alpha_{F}^{1}+x_{F}^{2}+X_{L}$ and $\Phi^{2}\left(\mathbf{x}_{F}, \mathbf{x}_{L}\right) \equiv$ $x_{F}^{2}-1+\alpha_{F}^{2}+x_{F}^{1}+X_{L}$. We deduce:

$$
\left|\mathcal{J}_{\mathbf{\Phi}_{\mathbf{x}_{F}}}\left(\mathbf{x}_{F}, \mathbf{x}_{L}\right)\right|=\left|\begin{array}{ll}
1 & 1 \\
1 & 1
\end{array}\right|=0 .
$$

As $\alpha_{F}^{1} \neq \alpha_{F}^{2}$ there is no solution to (27)-(28). Then, if $\left|\mathcal{J}_{\boldsymbol{\Phi}}\left(\mathbf{x}_{F}, \mathbf{x}_{L}\right)\right|=0$, then $\varphi_{L}^{j}\left(\mathbf{x}_{L}\right)=\{\varnothing\}, j=1,2$. We remark that $(2 \mathrm{~b})$ is not satisfied since:

$$
\frac{d^{2} c_{F}^{j}\left(x_{F}^{j}\right)}{\left(d x_{F}^{j}\right)^{2}}=-1, \text { which is not such that }-1>-1, j=1,2 .
$$

The nonexistence stems here from the presence of increasing returns to scale. 


\subsection{Nonuniqueness}

The inverse market demand is given by:

$$
p(X)=\frac{1}{\sqrt{X-1}},
$$

where $\lim _{X \rightarrow 1^{+}} p(X)=\infty, \lim _{X \rightarrow \infty} p(X)=0, \frac{d p(X)}{d X}=-\frac{1}{2}(X-1)^{-\frac{3}{2}}$ and $\frac{d^{2} p(X)}{(d X)^{2}}=$ $-\frac{3}{4}(X-1)^{-\frac{5}{2}}$. Let $c_{L}^{i}\left(x_{L}^{i}\right)=\sqrt{3} x_{L}^{i}, i=1,2$, and $c_{F}^{j}\left(x_{F}^{j}\right)=0, j=1,2$. Let $X^{*}=p^{-1}(\max \{\sqrt{3}, 0\})=\sqrt{3}$, so $x_{L}^{i} \in[0, \sqrt{3}], i=1,2$, and $x_{F}^{j} \in[0, \sqrt{3}], j=1,2$.

Followers' optimal decisions are given by:

$$
\begin{gathered}
\phi^{1}\left(x_{F}^{2}, \mathbf{x}_{L}\right)=2-2 x_{F}^{2}-2 X_{L} \\
\phi^{2}\left(x_{F}^{1}, \mathbf{x}_{L}\right)=2-2 x_{F}^{1}-2 X_{L} .
\end{gathered}
$$

Using (5), we deduce:

$$
\left|\mathcal{J}_{\mathbf{\Phi}_{\mathbf{x}_{F}}}\left(\mathbf{x}_{F}, \mathbf{x}_{L}\right)\right|=4\left|\begin{array}{ll}
1 & 1 \\
1 & 1
\end{array}\right|=0 \text {. }
$$

We deduce $\varphi^{j}\left(\mathbf{x}_{L}\right)=\frac{2}{3}-\frac{2}{3} X_{L}, j=1,2$. Then, $\nu=-\frac{4}{3}$ whenever $x_{L}^{i}>0$, $i=1,2$. Thus, using (32)-(33), leader $i$ solves $x_{L}^{i} \in \max \sqrt{\frac{3}{1-X_{L}}}-\sqrt{3} x_{L}^{i}, i=$ 1,2 . There are two symmetric equilibria $\left(\tilde{\mathbf{x}}_{L}, \tilde{\mathbf{x}}_{F}\right)=\left((0,0),\left(\frac{2}{3}, \frac{2}{3}\right)\right)$ and $\left(\tilde{\mathbf{x}}_{L}, \tilde{\mathbf{x}}_{F}\right)=$ $\left(\left(\frac{4}{9}, \frac{4}{9}\right),\left(\frac{2}{27}, \frac{2}{27}\right)\right)$. In the former $\nu=0$, so we have:

$$
\begin{aligned}
\frac{d p(X)}{d X}+\tilde{x}_{L}^{i} \frac{d^{2} p(X)}{(d X)^{2}} & =-\frac{3 \sqrt{3}}{2}<0 \\
\frac{d^{2} c_{L}^{i}\left(x_{L}^{i}\right)}{\left(d x_{L}^{i}\right)^{2}} & =0>-\frac{3 \sqrt{3}}{2}=\frac{d p(X)}{d X}, i=1,2
\end{aligned}
$$

and

$$
\begin{aligned}
\frac{d p(X)}{d X}+\tilde{x}_{F}^{j} \frac{d^{2} p(X)}{(d X)^{2}} & =3 \sqrt{3}>0 \\
\frac{d^{2} c_{F}^{j}\left(x_{F}^{j}\right)}{\left(d x_{F}^{j}\right)^{2}} & =0>-\frac{3 \sqrt{3}}{2}=\frac{d p(X)}{d X}, j=1,2,
\end{aligned}
$$

while in the latter we get:

$$
\begin{aligned}
\frac{d p(X)}{d X}+(1+\nu) \tilde{x}_{L}^{i} \frac{d^{2} p(X)}{(d X)^{2}} & =\frac{405}{2}>0 \\
\frac{d^{2} c_{L}^{i}\left(x_{L}^{i}\right)}{\left(d x_{L}^{i}\right)^{2}} & =0<\frac{27 \sqrt{3}}{2}=(1+\nu) \frac{d p(X)}{d X}, i=1,2
\end{aligned}
$$

and

$$
\frac{d p(X)}{d X}+\tilde{x}_{F}^{j} \frac{d^{2} p(X)}{(d X)^{2}}=81 \sqrt{3}>0
$$

$$
\frac{d^{2} c_{F}^{j}\left(x_{F}^{j}\right)}{\left(d x_{F}^{j}\right)^{2}}=0<81 \sqrt{3}=\frac{d p(X)}{d X}, j=1,2 .
$$

Finally, we have sign $\left|J_{-\Pi_{L}}\left((0,0),\left(\frac{2}{3}, \frac{2}{3}\right)\right)\right|<0$, sign $\left|J_{-\Pi_{F}}\left((0,0),\left(\frac{2}{3}, \frac{2}{3}\right)\right)\right|<0$, sign $\left|J_{-\Pi_{L}}\left(\left(\frac{4}{9}, \frac{4}{9}\right),\left(\frac{2}{27}, \frac{2}{27}\right)\right)\right|<0$, and sign $\left|J_{-\Pi_{F}}\left(\left(\frac{4}{9}, \frac{4}{9}\right),\left(\frac{2}{27}, \frac{2}{27}\right)\right)\right|>0$. 


\section{CONCLUSION}

The paper provides new proofs for the existence and uniqueness of SE in the multiple leader-follower model. Our noncooperative oligopoly model embodies two partial simultaneous move games with heterogeneous firms. As a hierarchical model, it consists of two Cournot competitions encompassed by a Stackelberg competition. One salient feature is the existence of a Nash equilibrium for the entire game is also based on consistent optimal decisions of followers. Our Lemma 2 provides a criterion to test for the existence of best responses, and thereby for the existence of a SE. In addition, our model generalizes some models of the literature as it displays some heterogeneity among firms which may have different costs, some of which may be nonconvex.

The main conclusions of the paper may be stated as follows. First, failure of existence stems from the fact that the system of equations that implicitly defines the best responses is inconsistent. Under Assumptions 1 and 2 the system is always consistent. Second, uniqueness is ensured since best responses are decreasing functions with negative slopes strictly greater than minus unity.

These results suggest three final comments. First, our model embodies two step of decisions and should be developed to embody a finite number of steps larger than two. Second, we should consider the strategic complementarities at work when best responses are increasing. Third, since strategic complementarities affect payoffs, the endogeneization of the order of moves should be undertaken within this framework.

\section{APPENDIX}

The proofs bring into light the role played by Assumptions 1 and 2, and more specifically, which parts in the two Assumptions play a critical role.

\subsection{Appendix A: Proof of Proposition 2}

The Jacobian matrix $\mathcal{J}_{\phi_{\mathbf{x}_{F}}}\left(\overline{\mathbf{x}}_{F}, \overline{\mathbf{x}}_{L}\right)$ is bounded. Differentiating partially the identity $\frac{\partial \pi^{j}}{\partial x_{F}^{j}}\left(\phi^{j}\left(\mathbf{x}_{F}^{-j}, \mathbf{x}_{L}\right), \mathbf{x}_{F}^{-j}, \mathbf{x}_{L}\right) \equiv 0$ with respect to $x_{F}^{-j}$, we get:

$$
\frac{\partial \phi^{j}}{\partial x_{F}^{-j}}=-\frac{\frac{\partial^{2} \pi_{F}^{j}}{\partial x_{F}^{j} \partial x_{F}^{-j}}}{\frac{\partial^{2} \pi_{F}^{j}}{\partial\left(x_{F}^{j}\right)^{2}}}=-\frac{\frac{d p(X)}{d X}+x_{F}^{j} \frac{d^{2} p(X)}{(d X)^{2}}}{2 \frac{d p(X)}{d X}+x_{F}^{j} \frac{d^{2} p(X)}{(d X)^{2}}-\frac{d^{2} c_{F}^{j}\left(x_{F}^{j}\right)}{\left(d x_{F}^{j}\right)^{2}}}
$$

From (1b)-(1c) and (2b), we get $\frac{\partial \phi^{j}}{\partial x_{F}^{-j}} \in(-1,0)$, when $\phi^{j}>0$, and $\frac{\partial \phi^{j}}{\partial x_{F}^{-j}}=$ 0 when $\phi^{j}=0$. Then, $\frac{\partial \phi^{j}}{\partial x_{F}^{-j}} \in(-1,0],-j, j \in \mathcal{F}_{F}$. So, the diagonal terms of $\mathcal{J}_{\phi_{\mathbf{x}_{F}}}\left(\overline{\mathbf{x}}_{F}, \overline{\mathbf{x}}_{L}\right)$ consist of zeros and the off-diagonal terms are negative and above -1 .

The Jacobian matrix $\mathcal{J}_{\phi_{\mathbf{x}_{L}}}\left(\overline{\mathbf{x}}_{F}, \overline{\mathbf{x}}_{L}\right)$ is bounded. Let $x_{F}^{j}=\phi^{j}\left(\mathbf{x}_{F}^{-j}, \mathbf{x}_{L}\right) \in\left(0, X^{*}\right)$, $j \in \mathcal{F}_{F}$ and write (4) as an identity:

$$
p(X)+x_{F}^{j}\left(\mathbf{x}_{F}^{-j}, \mathbf{x}_{L}\right) \frac{d p(X)}{d X}-\frac{d c_{F}^{j}\left(x_{F}^{j}\left(\mathbf{x}_{F}^{-j}, \mathbf{x}_{L}\right)\right)}{d x_{F}^{j}} \equiv 0,
$$

with $X=\phi^{1}\left(\mathbf{x}_{F}^{-1}, \mathbf{x}_{L}\right)+\ldots+\phi^{j}\left(\mathbf{x}_{F}^{-j}, \mathbf{x}_{L}\right)+\ldots+\sum_{i} x_{L}^{i}$. Differentiating partially with respect to $x_{L}^{i}$ yields, after rearrangement: 


$$
\left(\sum_{j} \frac{\partial \phi^{j}}{\partial x_{L}^{i}}+1\right)\left(\frac{d p(X)}{d X}+x_{F}^{j} \frac{d^{2} p(X)}{(d X)^{2}}\right)+\left(\frac{d p(X)}{d X}-\frac{d^{2} c_{F}^{j}\left(x_{F}^{j}\right)}{\left(d x_{F}^{j}\right)^{2}}\right) \frac{\partial \phi^{j}}{\partial x_{L}^{i}} \equiv 0
$$

This indentity is true only if $\left(\sum_{j} \frac{\partial \phi^{j}}{\partial x_{L}^{i}}+1\right) \frac{\partial \phi^{j}}{\partial x_{L}^{i}} \leqslant 0$ under $(1 \mathrm{~b})$ and (2b). In addition, if $\frac{\partial \phi^{j}}{\partial x_{L}^{i}} \geqslant 0$, then $\frac{\partial \phi^{j}}{\partial x_{L}^{i}}+\sum_{-j \neq j} \frac{\partial \phi^{-j}}{\partial x_{L}^{i}}+1 \leqslant 0$, a contradiction. Then $\frac{\partial \phi^{j}}{\partial x_{L}^{i}} \leqslant 0$, with $\sum_{j} \frac{\partial \phi^{j}}{\partial x_{L}^{i}}>-1$. Then, we conclude $\frac{\partial \phi^{j}}{\partial x_{L}^{i}} \in(-1,0], i \in \mathcal{F}_{L}, j \in \mathcal{F}_{F}$.

\subsection{Appendix B: Proof of Lemma 2}

We show (5) has a solution, namely $\mathbf{x}_{F}=\varphi\left(\mathbf{x}_{L}\right)$, with component function $x_{F}^{j}=\varphi^{j}\left(\mathbf{x}_{L}\right), j \in \mathcal{F}_{F}$. Consider $\boldsymbol{\Phi}\left(\mathbf{x}_{F}, \mathbf{x}_{L}\right)=\left(\Phi^{1}\left(\mathbf{x}_{F}, \mathbf{x}_{L}\right), \ldots, \Phi^{n_{F}}\left(\mathbf{x}_{F}, \mathbf{x}_{L}\right)\right)$. Since we focus on inner solutions, consider the restriction of $\prod_{j} \mathcal{S}_{F}^{j} \times \prod_{i} \mathcal{S}_{L}^{i}$ to the open set $\prod_{j} \overline{\mathcal{S}}_{F}^{j} \times \prod_{i} \overline{\mathcal{S}}_{L}^{i}$, with $\overline{\mathcal{S}}_{L}^{i} \subset \mathcal{S}_{L}^{i}, i \in \mathcal{F}_{L}$ and $\overline{\mathcal{S}}_{F}^{j} \subset \mathcal{S}_{F}^{j}, j \in \mathcal{F}_{F}$. The vector function $\mathbf{\Phi}\left(\mathbf{x}_{F}, \mathbf{x}_{L}\right)$ is $C^{1}$ on the open set $\prod_{j} \overline{\mathcal{S}}_{F}^{j} \times \prod_{i} \overline{\mathcal{S}}_{L}^{i}$ as each $\Phi^{j}$ is a $C^{1}$ function of $\left(\mathbf{x}_{F}, \mathbf{x}_{L}\right)$ on the open set $\prod_{j} \overline{\mathcal{S}}_{F}^{j} \times \prod_{i} \overline{\mathcal{S}}_{L}^{i}$. Consider the $n_{F}$-dimensional vector equation $\mathbf{\Phi}\left(\mathbf{x}_{F}, \mathbf{x}_{L}\right)=\mathbf{0}$. Let $\left(\overline{\mathbf{x}}_{F}, \overline{\mathbf{x}}_{L}\right)$ be an interior point of $\prod_{j} \overline{\mathcal{S}}_{F}^{j} \times \prod_{i} \overline{\mathcal{S}}_{L}^{i}$, where $\overline{\mathbf{x}}_{L}$ corresponds to a parameter configuration, with $\overline{\mathbf{x}}_{L} \in \prod_{i} \overline{\mathcal{S}}_{L}^{i}$, so we have the identity $\boldsymbol{\Phi}\left(\mathbf{x}_{F}\left(\mathbf{x}_{L}\right), \mathbf{x}_{L}\right) \equiv \mathbf{0}$ in an open neighborhood of $\left(\overline{\mathbf{x}}_{F}, \overline{\mathbf{x}}_{L}\right)$. Implicit partial differentiation with respect to $\mathbf{x}_{L}$ of this identity leads to:

$\mathcal{J}_{\Phi_{\mathbf{x}_{F}}}\left(\overline{\mathbf{x}}_{F}, \overline{\mathbf{x}}_{L}\right) . \mathbf{A}=-\mathbf{B}$
where $\mathcal{J}_{\boldsymbol{\Phi}_{\mathbf{x}_{F}}}\left(\overline{\mathbf{x}}_{F}, \overline{\mathbf{x}}_{L}\right)=\left[\begin{array}{lllll}1 & \ldots & \frac{\partial \Phi^{1}}{\partial x_{F}^{j}} & \ldots & \frac{\partial \Phi^{1}}{\partial x_{F}^{n_{F}}} \\ \ldots & \ldots & \ldots & \ldots & \ldots \\ \frac{\partial \Phi^{j}}{\partial x_{F}^{1}} & \ldots & 1 & \ldots & \frac{\partial \Phi^{j}}{\partial x_{F}^{n_{F}}} \\ \ldots & \ldots & \ldots & \ldots & \ldots \\ \frac{\partial \Phi^{n_{F}}}{\partial x_{F}^{1}} & \ldots & \frac{\partial \Phi^{n_{F}}}{\partial x_{F}^{j}} & \ldots & 1\end{array}\right]$ is a $\left(n_{F}, n_{F}\right)$ matrix, and

$\mathbf{A} \equiv\left[\begin{array}{ccccc}\frac{\partial x_{F}^{1}}{\partial x_{L}^{1}} & \ldots & \frac{\partial x_{F}^{1}}{\partial x_{L}^{i}} & \ldots & \frac{\partial x_{F}^{1}}{\partial x_{L}^{n} L} \\ \ldots & \ldots & \ldots & \ldots & \ldots \\ \frac{\partial x_{F}^{j}}{\partial x_{L}^{1}} & \ldots & \frac{\partial x_{F}^{j}}{\partial x_{L}^{i}} & \ldots & \frac{\partial x_{F}^{j}}{\partial x_{L}^{n_{L}}} \\ \ldots & \ldots & \ldots & \ldots & \ldots \\ \frac{\partial x_{F}^{n_{F}}}{\partial x_{L}^{1}} & \ldots & \frac{\partial x_{F}^{n}}{\partial x_{L}^{i}} & \ldots & \frac{\partial x_{F}^{n_{F}}}{\partial x_{L}^{n_{L}}}\end{array}\right]$ and $\mathbf{B} \equiv\left[\begin{array}{ccccc}\frac{\partial \Phi^{1}}{\partial x_{L}^{1}} & \ldots & \frac{\partial \Phi^{1}}{\partial x_{L}^{i}} & \ldots & \frac{\partial \Phi^{1}}{\partial x_{L}^{n_{L}}} \\ \ldots & \ldots & \ldots & \ldots & \ldots \\ \frac{\partial \Phi^{j}}{\partial x_{L}^{1}} & \ldots & \frac{\partial \Phi^{j}}{\partial x_{L}^{i}} & \ldots & \frac{\partial \Phi^{j}}{\partial x_{L}^{n_{L}}} \\ \ldots & \ldots & \ldots & \ldots & \ldots \\ \frac{\partial \Phi^{n} F}{\partial x_{L}^{1}} & \ldots & \frac{\partial \Phi^{n} F}{\partial x_{L}^{i}} & \ldots & \frac{\partial \Phi^{n}}{\partial x_{L}^{n_{L}}}\end{array}\right]$

are matrices of dimension $\left(n_{F}, n_{L}\right)$ respectively.

We first show that $\mathcal{J}_{\boldsymbol{\Phi}_{\mathbf{x}_{F}}}\left(\overline{\mathbf{x}}_{F}, \overline{\mathbf{x}}_{L}\right)$ is a strictly positive square matrix. From (5), for each $j \in \mathcal{F}_{F}$, we have $\frac{\partial \Phi^{j}}{\partial x_{F}^{-j}}=-\frac{\partial \phi^{j}}{\partial x_{F}^{-j}},-j \neq j$. And, from Proposition 2, we know that $\mathcal{J}_{\phi_{\mathbf{x}_{F}}}\left(\overline{\mathbf{x}}_{F}, \overline{\mathbf{x}}_{L}\right) \in\left(-\mathcal{I}_{\left(n_{F}, n_{F}\right)}, \mathbf{0}_{\left(n_{F}, n_{F}\right)}\right]$, which, from (5), means 
$\frac{\partial \Phi^{j}}{\partial x_{F}^{-j}} \in[0,1),-j \neq j,-j, j \in \mathcal{F}_{F}$. Then, we deduce $\mathbf{0}_{\left(n_{F}, n_{F}\right)} \leq-\mathcal{J}_{\phi_{\mathbf{x}_{F}}}\left(\overline{\mathbf{x}}_{F}, \overline{\mathbf{x}}_{L}\right)<<$ $\mathcal{I}_{\left(n_{F}, n_{F}\right)}$. Therefore, we have $\mathcal{J}_{\boldsymbol{\Phi}_{\mathbf{x}_{F}}}\left(\overline{\mathbf{x}}_{F}, \overline{\mathbf{x}}_{L}\right)=\mathbf{I}-\mathcal{J}_{\phi_{\mathbf{x}_{F}}}\left(\overline{\mathbf{x}}_{F}, \overline{\mathbf{x}}_{L}\right)$, where $\mathbf{I}$ is the identity matrix of dimension $\left(n_{F}, n_{F}\right)$. So, the matrix $\mathcal{J}_{\mathbf{\Phi}_{\mathbf{x}_{F}}}\left(\overline{\mathbf{x}}_{F}, \overline{\mathbf{x}}_{L}\right)$ has unit terms on the main diagonal and strictly positive (less than one) off-diagonal terms. We deduce $\mathcal{J}_{\boldsymbol{\Phi}_{\mathbf{x}_{F}}}\left(\overline{\mathbf{x}}_{F}, \overline{\mathbf{x}}_{L}\right)>>\mathbf{0}_{\left(n_{F}, n_{F}\right)}$. But then, the matrix $\mathcal{J}_{\boldsymbol{\Phi}_{\mathbf{x}_{F}}}\left(\overline{\mathbf{x}}_{F}, \overline{\mathbf{x}}_{L}\right)$ has strictly positive real eigenvalues $e^{j}, j=1, \ldots, n_{F}$. As the determinant is the product of such eigenvalues, i.e., $\left|\mathcal{J}_{\boldsymbol{\Phi}_{\mathbf{x}_{F}}}\left(\overline{\mathbf{x}}_{F}, \overline{\mathbf{x}}_{L}\right)\right|=\prod_{j} e^{j}>0$, we conclude $\left|\mathcal{J}_{\boldsymbol{\Phi}_{\mathbf{x}_{F}}}\left(\overline{\mathbf{x}}_{F}, \overline{\mathbf{x}}_{L}\right)\right|>0$. Then, by the Implicit Function Theorem, there exist open sets $\mathcal{U}$ in $\prod_{j} \bar{S}_{F}^{j} \times \prod_{i} \overline{\mathcal{S}}_{L}^{i}$ and $\mathcal{U}_{\left(\mathbf{x}_{L}\right)}$ in $\prod_{i} \bar{S}_{L}^{i}$, with $\left(\overline{\mathbf{x}}_{F}, \overline{\mathbf{x}}_{L}\right) \subseteq \mathcal{U}$ and $\overline{\mathbf{x}}_{L} \subseteq \mathcal{U}_{\left(\mathbf{x}_{L}\right)}$ such that for each $\mathbf{x}_{L}$ in $\mathcal{U}_{\left(\mathbf{x}_{L}\right)}$, there exists a locally unique $n_{F}$ dimensional vector function $\mathbf{x}_{F}\left(\mathbf{x}_{L}\right)$ in some neighborhood of $\left(\overline{\mathbf{x}}_{F}, \overline{\mathbf{x}}_{L}\right)$ such that $\left(\mathbf{x}_{F}\left(\mathbf{x}_{L}\right), \mathbf{x}_{L}\right) \in \mathcal{U}$ and $\mathbf{\Phi}\left(\mathbf{x}_{F}\left(\mathbf{x}_{L}\right), \mathbf{x}_{L}\right) \equiv \mathbf{0}$. The solution $\mathbf{x}_{F}\left(\mathbf{x}_{L}\right)=\mathbf{\Phi}^{-1}(\mathbf{0})$ is denoted by $\boldsymbol{\varphi}\left(\mathbf{x}_{L}\right)$, and is defined by $\boldsymbol{\varphi}: \prod_{i} \mathcal{S}_{L}^{i} \supset$ $\mathcal{U}_{\left(\overline{\mathbf{x}}_{L}\right)} \rightarrow \prod_{j} \mathcal{S}_{F}^{j}$, with $\mathbf{x}_{F}=\varphi\left(\mathbf{x}_{L}\right)$. Each component function $\varphi^{j}\left(\mathbf{x}_{L}\right)$ is defined as $\varphi^{j}: \prod_{i} \mathcal{S}_{L}^{i} \supset \mathcal{U}_{\left(\overline{\mathbf{x}}_{L}\right)} \rightarrow \mathcal{S}_{F}^{j}$, with $x_{F}^{j}=\varphi^{j}\left(\mathbf{x}_{L}\right), j \in \mathcal{F}_{F}$. In addition, the function $\boldsymbol{\varphi}\left(\mathbf{x}_{L}\right)$ is continuously differentiable. Then, for each $j \in \mathcal{F}_{F}, \varphi^{j}\left(\mathbf{x}_{L}\right)$ is $C^{1}$.

\subsection{Appendix C: Proof of Proposition 3}

We must show that for all $i \in \mathcal{F}_{L},-1<\frac{\partial \varphi^{j}}{\partial x_{L}^{i}} \leqslant 0, j \in \mathcal{F}_{F}$. We first show $\frac{\partial \varphi^{j}}{\partial x_{L}^{i}} \leqslant 0, j \in \mathcal{F}_{F}$. Consider the system given by (6), and let $\frac{\partial x_{F}^{1}}{\partial x_{L}^{-i}}=0$ for all $-i \neq i$ in $\mathbf{A}$ and $\frac{\partial \Phi^{j}}{\partial x_{L}^{-i}}=0$ for all $-i \neq i$ in $\mathbf{B}$. Then, from Cramer's rule, we deduce:

$$
\frac{\partial x_{F}^{j}}{\partial x_{L}^{i}}=-\frac{\left|\mathcal{J}_{\boldsymbol{\Phi}_{\mathbf{x}_{F}}}^{\prime}\left(\overline{\mathbf{x}}_{F}, \overline{\mathbf{x}}_{L}\right)\right|}{\left|\mathcal{J}_{\boldsymbol{\Phi}_{\mathbf{x}_{F}}}\left(\overline{\mathbf{x}}_{F}, \overline{\mathbf{x}}_{L}\right)\right|},
$$

where $\mathcal{J}_{\mathbf{\Phi}_{\mathbf{x}_{F}}}^{\prime}\left(\overline{\mathbf{x}}_{F}, \overline{\mathbf{x}}_{L}\right)$ is the $\left(n_{F}, n_{F}\right)$ square matrix obtained by replacing the $j$ th column in $\mathcal{J}_{\boldsymbol{\Phi}}\left(\overline{\mathbf{x}}_{F}, \overline{\mathbf{x}}_{L}\right)$ by the $i$ th positive member of $\mathbf{B}$, so that:

$$
\mathcal{J}_{\mathbf{\Phi}_{\mathbf{x}_{F}}}^{\prime}\left(\overline{\mathbf{x}}_{F}, \overline{\mathbf{x}}_{L}\right)=\left[\begin{array}{lllll}
1 & \ldots & \frac{\partial \Phi^{1}}{\partial x_{L}^{i}} & \ldots & \frac{\partial \Phi^{1}}{\partial x_{F}^{n_{F}}} \\
\ldots & \ldots & \ldots & \ldots & \ldots \\
\frac{\partial \Phi^{j}}{\partial x_{F}^{1}} & \ldots & \frac{\partial \Phi^{j}}{\partial x_{L}^{i}} & \ldots & \frac{\partial \Phi^{j}}{\partial x_{F}^{n}} \\
\ldots & \ldots & \ldots & \ldots & \ldots \\
\frac{\partial \Phi^{n} F}{\partial x_{F}^{1}} & \ldots & \frac{\partial \Phi^{n}}{\partial x_{L}^{i}} & \ldots & 1
\end{array}\right] .
$$

We know $\left|\mathcal{J}_{\mathbf{\Phi}_{\mathbf{x}_{F}}}\left(\overline{\mathbf{x}}_{F}, \overline{\mathbf{x}}_{L}\right)\right|>0$, so we deduce:

$$
\operatorname{sign} \frac{\partial x_{F}^{j}}{\partial x_{L}^{i}} \equiv \operatorname{sign}\left|\mathcal{J}_{\mathbf{\Phi}_{\mathbf{x}_{F}}}^{\prime}\left(\overline{\mathbf{x}}_{F}, \overline{\mathbf{x}}_{L}\right)\right|
$$

By definition of $p(X)$, for each $j \in \mathcal{F}_{F}$, we know that $\frac{\partial \Phi^{j}}{\partial x_{L}^{i}}=\frac{\partial \Phi^{j}}{\partial x_{L}^{-i}}=\frac{\partial \Phi^{j}}{\partial x_{F}^{-j}}$, $-i, i \in \mathcal{F}_{L},-j \in \mathcal{F}_{F}$. In addition, from Proposition 2, we have for all $j \in \mathcal{F}_{F}$, $\frac{\partial \phi^{j}}{\partial x_{L}^{i}} \in(-1,0], i \in \mathcal{F}_{L}$, and $\frac{\partial \phi^{j}}{\partial x_{F}^{-j}} \in(-1,0]$, for all $-j \neq j,-j \in \mathcal{F}_{F}$. Then, using (5) 
we deduce that for all $j \in \mathcal{F}_{F}, \frac{\partial \Phi^{j}}{\partial x_{F}^{-j}} \in[0,1),-j \in \mathcal{F}_{F}$, and $\frac{\partial \Phi^{j}}{\partial x_{L}^{i}} \in[0,1), i \in \mathcal{F}_{L}$. Then, the square matrix $\mathcal{J}_{\mathbf{\Phi}_{\mathbf{x}_{F}}}^{\prime}$ is nonnegative. Assume the $j$ th line is such that $\frac{\partial \Phi^{j}}{\partial x_{L}^{i}}=0, i \in \mathcal{F}_{L}$, that is, $\frac{\partial \Phi^{j}}{\partial x_{F}^{-j}}=0,-j \in \mathcal{F}_{F}$. Then, $\left|\mathcal{J}_{\Phi_{\mathbf{x}_{F}}}^{\prime}\left(\overline{\mathbf{x}}_{F}, \overline{\mathbf{x}}_{L}\right)\right|=0$, so $\frac{\partial x_{F}^{j}}{\partial x_{L}^{i}}=0, i \in \mathcal{F}_{L}$. Assume now the $j$ th line is such that $\frac{\partial \Phi^{j}}{\partial x_{L}^{i}} \in(0,1), i \in \mathcal{F}_{L}$. In addition, as $\frac{\partial \Phi^{-j}}{\partial x_{L}^{i}}=\frac{\partial \Phi^{-j}}{\partial x_{F}^{j}}$ for each line $-j \neq j$, we have $1>\sum_{j} \frac{\partial \Phi^{-j}}{\partial x_{F}^{j}}$. Therefore, there is some $\mathbf{q}>>\mathbf{0}$ such that $\frac{\partial \Phi^{j}}{\partial x_{F}^{i}}>0$ and for all $j \in \mathcal{F}_{F}, \sum_{i} q_{i}\left|\frac{\partial \Phi^{j}}{\partial x_{L}^{i}}\right|>0$, as we have $0<\sum_{j} \frac{\partial \Phi^{j}}{\partial x_{L}^{i}}<1$, and since for line $j$, $\frac{\partial \Phi^{j}}{\partial x_{L}^{i}}>0$, with $\sum_{j} \frac{\partial \Phi^{j}}{\partial x_{L}^{i}}<1$. Then, the square matrix $\mathcal{J}_{\mathbf{\Phi}_{\mathbf{x}_{F}}}^{\prime}\left(\overline{\mathbf{x}}_{F}, \overline{\mathbf{x}}_{L}\right)$ has a positive dominant diagonal. Then, $\left|\mathcal{J}_{\boldsymbol{\Phi}_{\mathbf{x}_{F}}}^{\prime}\left(\overline{\mathbf{x}}_{F}, \overline{\mathbf{x}}_{L}\right)\right|>0$. By repeting the argument for all $j \in \mathcal{F}_{F}$, from what preceeds, we deduce $\frac{\partial x_{F}^{j}}{\partial x_{L}^{i}}<0$, which means $\frac{\partial \varphi^{j}}{\partial x_{L}^{i}}<0, i \in \mathcal{F}_{L}, j \in \mathcal{F}_{F}$. Collecting both cases, we conclude $\frac{\partial \varphi^{j}}{\partial x_{L}^{i}} \leqslant 0, i \in \mathcal{F}_{L}, j \in \mathcal{F}_{F}$.

We now show for all $i \in \mathcal{F}_{L}, \frac{\partial \varphi^{j}}{\partial x_{L}^{i}}>-1, j \in \mathcal{F}_{F}$. Select one firm $j \in \mathcal{F}_{F}$. From what preceed, we know that:

$$
\frac{\partial \varphi_{F}^{j}}{\partial x_{L}^{i}}=-\frac{\left|\mathcal{J}_{\boldsymbol{\Phi}_{\mathbf{x}_{F}}}^{\prime}\left(\overline{\mathbf{x}}_{F}, \overline{\mathbf{x}}_{L}\right)\right|}{\left|\mathcal{J}_{\boldsymbol{\Phi}_{\mathbf{x}_{F}}}\left(\overline{\mathbf{x}}_{F}, \overline{\mathbf{x}}_{L}\right)\right|}, i \in \mathcal{F}_{L} .
$$

We also know that $\mathcal{J}_{\Phi_{\mathbf{x}_{F}}}^{\prime}\left(\overline{\mathbf{x}}_{F}, \overline{\mathbf{x}}_{L}\right)$ and $\mathcal{J}_{\Phi_{\mathbf{x}_{F}}}\left(\overline{\mathbf{x}}_{F}, \overline{\mathbf{x}}_{L}\right)$ have common terms since for each $-j \neq j,-j \in \mathcal{F}_{F}, \frac{\partial \Phi^{-j}}{\partial x_{L}^{i}}=\frac{\partial \Phi^{-j}}{\partial x_{F}^{j}}, i \in \mathcal{F}_{L}, j \in \mathcal{F}_{F}$. Assume $\frac{\partial \varphi_{F}^{j}}{\partial x_{L}^{i}}<-1$. It implies:

which leads to:

$$
-\frac{\left|\mathcal{J}_{\Phi_{\mathbf{x}_{F}}}^{\prime}\left(\overline{\mathbf{x}}_{F}, \overline{\mathbf{x}}_{L}\right)\right|}{\left|\mathcal{J}_{\boldsymbol{\Phi}_{\mathbf{x}_{F}}}\left(\overline{\mathbf{x}}_{F}, \overline{\mathbf{x}}_{L}\right)\right|}<-1
$$

$$
\left|\mathcal{J}_{\mathbf{\Phi}_{\mathbf{x}_{F}}}^{\prime}\left(\overline{\mathbf{x}}_{F}, \overline{\mathbf{x}}_{L}\right)\right|>\left|\mathcal{J}_{\boldsymbol{\Phi}_{\mathbf{x}_{F}}}\left(\overline{\mathbf{x}}_{F}, \overline{\mathbf{x}}_{L}\right)\right|
$$

Expansion by cofactors of $\left|\mathcal{J}_{\mathbf{\Phi}_{\mathbf{x}_{F}}}^{\prime}\left(\overline{\mathbf{x}}_{F}, \overline{\mathbf{x}}_{L}\right)\right|>\left|\mathcal{J}_{\boldsymbol{\Phi}_{\mathbf{x}_{F}}}\left(\overline{\mathbf{x}}_{F}, \overline{\mathbf{x}}_{L}\right)\right|$ and cancellation among common terms on both sides lead to:

$$
\frac{\partial \varphi^{j}}{\partial x_{L}^{i}}(-1)^{2 j}\left|\left(\mathcal{J}_{\boldsymbol{\Phi}_{\mathbf{x}_{F}}}^{\prime}\right)_{\left(n_{F}-1, n_{F}-1\right)}(\overline{\mathbf{x}})\right|>(-1)^{2 j}\left|\left(\mathcal{J}_{\Phi_{\mathbf{x}_{F}}}\right)_{\left(n_{F}-1, n_{F}-1\right)}(\overline{\mathbf{x}})\right|,
$$

with $\overline{\mathbf{x}}=\left(\overline{\mathbf{x}}_{F}, \overline{\mathbf{x}}_{L}\right)$, and where $\left|\left(\mathcal{J}_{\boldsymbol{\Phi}_{\mathbf{x}_{F}}}\right)_{\left(n_{F}-1, n_{F}-1\right)}(\overline{\mathbf{x}})\right|\left(\operatorname{resp} .\left|\left(\mathcal{J}_{\boldsymbol{\Phi}_{\mathbf{x}_{F}}}^{\prime}\right)_{\left(n_{F}-1, n_{F}-1\right)}(\overline{\mathbf{x}})\right|\right)$ stands for the principal minor of order $\left(n_{F}-1, n_{F}-1\right)$ of $\mathcal{J}_{\mathbf{\Phi}_{\mathbf{x}_{F}}}(\overline{\mathbf{x}})\left(\operatorname{resp} . \mathcal{J}_{\mathbf{\Phi}_{\mathbf{x}_{F}}}^{\prime}(\overline{\mathbf{x}})\right)$. But $\left|\left(\mathcal{J}_{\Phi_{\mathbf{x}_{F}}}^{\prime}\right)_{\left(n_{F}-1, n_{F}-1\right)}(\overline{\mathbf{x}})\right|=\left|\left(\mathcal{J}_{\boldsymbol{\Phi}_{\mathbf{x}_{F}}}\right)_{\left(n_{F}-1, n_{F}-1\right)}(\overline{\mathbf{x}})\right|$ by construction. Then, we deduce $\frac{\partial \varphi^{j}}{\partial x_{L}^{i}}>1$, which is false as we assumed $\frac{\partial \varphi^{j}}{\partial x_{L}^{i}}>-1$. A contradiction. Then, we have $-\frac{\left|\mathcal{J}_{\mathbf{\Phi}_{F}}^{\prime}\left(\overline{\mathbf{x}}_{F}, \overline{\mathbf{x}}_{L}\right)\right|}{\left|\mathcal{J}_{\mathbf{\Phi}_{F}}\left(\overline{\mathbf{x}}_{F}, \overline{\mathbf{x}}_{L}\right)\right|}<1$, so we deduce $\frac{\partial \varphi^{j}}{\partial x_{L}^{i}}>-1$, for all $i \in \mathcal{F}_{L}, j \in \mathcal{F}_{F}$. 


\section{ACKNOWLEDGMENTS}

I am grateful to L. De Mesnard, M. Tidball and J. Vacksmann for their comments and remarks on a preliminary version of this paper previously entitled "On the Existence and uniqueness of a Stackelberg Market Equilibrium" (September 2015). A second version was entitled "On Stackelberg Market Equilibrium in the Multiple Leader-Follower Game" (January 2016). I also aknowledge the participants to the interdisciplinary Mathematics and Economics seminar AMIS in Paris Nanterre, and notably O. Renault for his remarks. The CNRS interdisciplinary Mathematics and Economics project MoMIS devoted to Strategic Interactions and Markets is also gratefully acknowledged.

\section{REFERENCES}

[1] Alj, A., Breton, M., Haurie, A., 1988. Sequential Stackelberg equilibria in twoperson games, Journal of Optimization Theory and Applications. 59, 71-97.

[2] Berge, C., 1963. Espaces Topologiques (Fonctions Multivoques), Dunod, Paris.

[3] Boyer, M., Moreaux, M., 1986. Perfect competition as the limit of a hierarchical market game. Economics Letters. 22, 115-118.

[4] Daughety, A., 2009. Introduction, purpose, and overview, in: Daughety, A. (Eds.), Cournot oligopoly. Characterization and applications. Cambridge University Press, New-York, pp. 3-44.

[5] DeMiguel, V., Xu, H., 2009. A stochastic multiple-leader Stackelberg model: analysis, computation, and application, Operations Research. 57, 1220-1235.

[6] De Wolf, D., Smeers, Y., 1997. A stochastic version of a Stackelberg-NashCournot equilibrium model, Management Science. 43, 190-197.

[7] Ehrenmann, A., 2004. Manifolds of multi-leader Cournot equilibria, Operations Research Letters. 32, 121-125.

[8] Frank, C.R., Quandt R.E., 1963. On the existence of a Cournot equilibrium, International Economic Review. 4, 92-96.

[9] Freiling, G., Jank, G., Lee, S.R., 2001. Existence and uniqueness of open loop Stackelberg equilibria in linear quadratic differential games, Journal of Optimization Theory and Applications. 110, 515-544.

[10] Fukushima, M., Pang, J.S., 2005. Quasi-variational inequalities, generalized Nash equilibria, and multi-leader-follower games, Computer Management Sciences. 2, 21-56.

[11] Hahn, F.H., 1962. The stability of the Cournot oligopoly solution, The Review of Economic Studies. 29, 329-333.

[12] He, J., Jia, J.W., Xiang, S., 2015. Existence and stability of weakly ParetoNash equilibrium for generalized multiobjective multi-leder-follower games, Journal of Global Optimization. 61, 397-405.

[13] Johansen, L., 1982. On the status of the Nash type of noncooperative equilibrium in economic theory, The Scandinavian Journal of Economics. 84, 421-441. 
[14] Julien, L.A., 2011. A note on Stackelberg competition, Journal of Economics. 103, 171-187.

[15] Kolstad, C.D., Mathiesen, L., 1987. Necessary and sufficient conditions for uniqueness of a Cournot equilibrium, The Review of Economic Studies. 54, 681-690.

[16] Murphy, F.H., Sherali, H.D., Soyster, A.L., 1983. Stackelberg-Nash-Cournot equilibria: characterizations and computations, Operations Research. 31, 253276.

[17] Novshek, W., 1985. On the existence of Cournot equilibrium, The Review of Economic Studies. 52, 85-98.

[18] Okuguchi, K., 1976. Expectations and Stability in Oligopoly Models. Lecture Notes in Economics and Mathematical Systems, Berlin, Heidelberg, New York.

[19] Robson, A.J., 1990. Stackelberg and Marshall, The American Economic Review. 80, 69-82.

[20] Selten, R., 1975. Re-examination of the perfectness concept for equilibrium points in extensive games, International Journal of Game Theory. 4, 25-55.

[21] Sherali, H.D., 1984. A multiple leader Stackelberg model and analysis, Operations Research. 32, 390-405.

[22] Tobin, R.L., 1992. Uniqueness results and algorithm for Stackelberg-CournotNash equilibria, Annals of Operations Research. 34, 21-36.

[23] Vives, X., 1999. Oligopoly pricing. Olds ideas and new tools, MIT Press, Cambridge 1999 .

[24] von Stackelberg, H., 1934. Marktform und Gleichgewicht, Springer, Berlin \& Vienna.

[25] Yu, J., Wang, H.L., 2008. An existence theorem for equilibrium points for multi-leader-follower games, Nonlinear Analysis. 69, 1775-1777. 